\title{
$\mathrm{BMC}$
}

Systems Biology

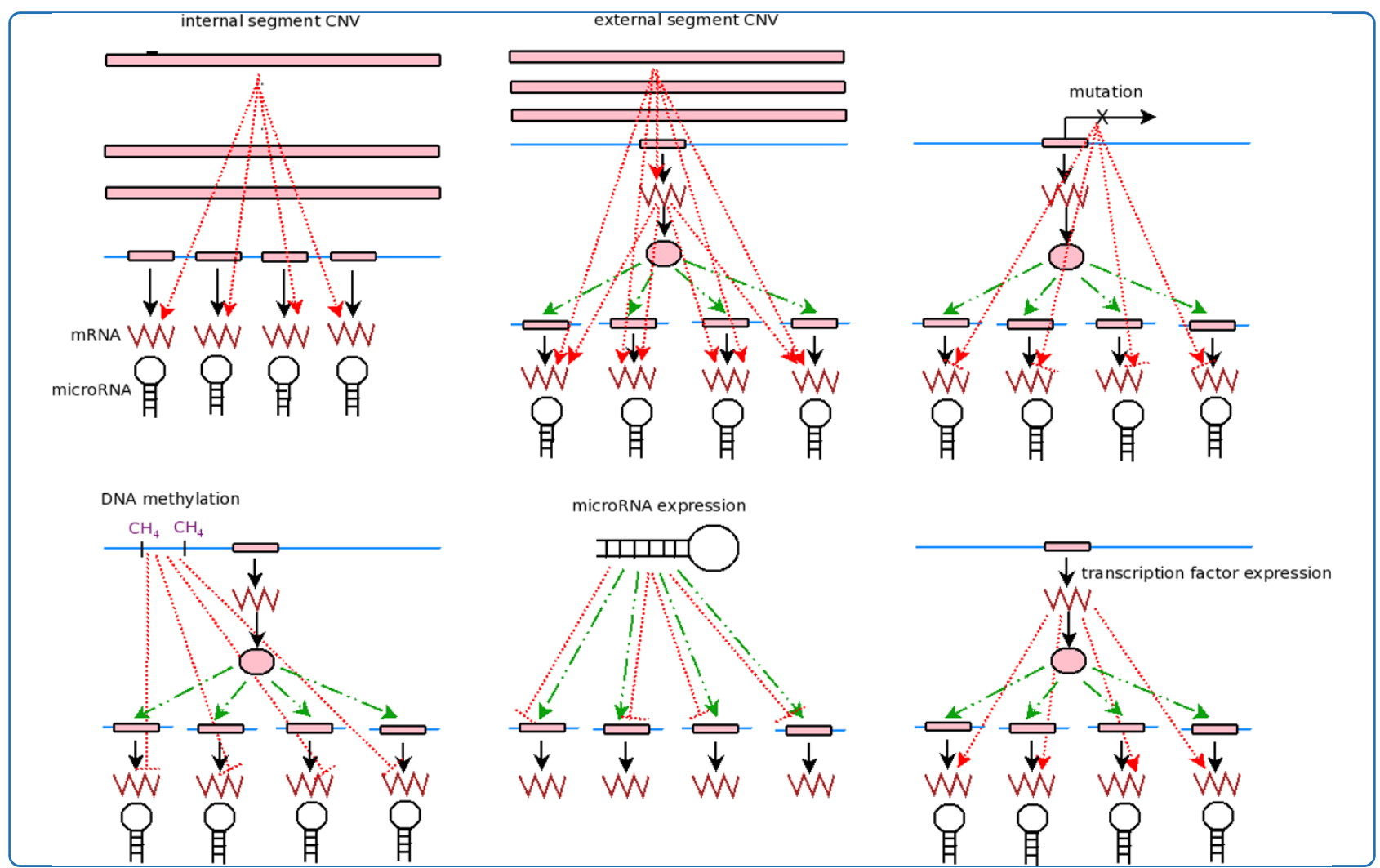

\section{Deciphering causal and statistical relations of molecular aberrations and gene expressions in $\mathrm{NCl}-60$ cell lines}

Li et al. 


\title{
Deciphering causal and statistical relations of molecular aberrations and gene expressions in $\mathrm{NCl}-60$ cell lines
}

\author{
Shyh-Dar Li ${ }^{2}$, Tatsuaki Tagami ${ }^{3}$, Ying-Fu Ho ${ }^{1}$ and Chen-Hsiang Yeang ${ }^{*}$
}

\begin{abstract}
Background: Cancer cells harbor a large number of molecular alterations such as mutations, amplifications and deletions on DNA sequences and epigenetic changes on DNA methylations. These aberrations may dysregulate gene expressions, which in turn drive the malignancy of tumors. Deciphering the causal and statistical relations of molecular aberrations and gene expressions is critical for understanding the molecular mechanisms of clinical phenotypes.

Results: In this work, we proposed a computational method to reconstruct association modules containing driver aberrations, passenger mRNA or microRNA expressions, and putative regulators that mediate the effects from drivers to passengers. By applying the module-finding algorithm to the integrated datasets of $\mathrm{NCl}-60$ cancer cell lines, we found that gene expressions were driven by diverse molecular aberrations including chromosomal segments' copy number variations, gene mutations and DNA methylations, microRNA expressions, and the expressions of transcription factors. In-silico validation indicated that passenger genes were enriched with the regulator binding motifs, functional categories or pathways where the drivers were involved, and co-citations with the driver/regulator genes. Moreover, 6 of 11 predicted MYB targets were down-regulated in an MYB-siRNA treated leukemia cell line. In addition, microRNA expressions were driven by distinct mechanisms from mRNA expressions.

Conclusions: The results provide rich mechanistic information regarding molecular aberrations and gene expressions in cancer genomes. This kind of integrative analysis will become an important tool for the diagnosis and treatment of cancer in the era of personalized medicine.
\end{abstract}

\section{Background}

Cancer cells harbor a large number of alterations at genetic, epigenetic and phenotypic levels. High-throughput screenings identified hundreds of somatic mutations (e.g., $[1,2])$, thousands of gene and microRNA expression changes (e.g., $[3,4])$ and copy number variations (e. g., [5]), large-scale epigenetic variations (e.g., [6]) in a typical cancer cell. Only a small fraction of these alterations may drive the malignancy of cancers, whereas the majority of them are likely the by-products of chromatin instability and dysregulation of transcriptional/translational apparatus. Separation of driver from passenger

\footnotetext{
* Correspondence: chyeang@webmail.stat.sinica.edu.tw 'Institute of Statistical Science, Academia Sinica, Academia Road, Sec 2, Taipei, Taiwan

Full list of author information is available at the end of the article
}

aberrations is a key question of cancer genomics for its strong implications in prognosis and treatments.

Finding the causal and mechanistic links connecting driver aberrations and clinical phenotypes is challenging due to the complexity of the underlying processes. Alternatively, molecular phenotypes such as gene expressions are considered. Genetic and epigenetic alterations on DNAs modulate the expressions and activities of key regulators such as transcription factors and microRNAs. Dysregulation of these molecules in turn affects a large number of downstream genes. The global gene expression and activity changes then influence clinical phenotypes such as proliferation rates, drug resistance and capability of metastasis.

Within the context of mechanisms we modify and extend the definitions of drivers and passengers. A driver is a molecular aberration (mutation, copy number
C Biomed Central

() 2011 Li et al; licensee BioMed Central Ltd. This is an Open Access article distributed under the terms of the Creative Commons Attribution License (http://creativecommons.org/licenses/by/2.0), which permits unrestricted use, distribution, and reproduction in any medium, provided the original work is properly cited. 
variation, DNA methylation, etc.) that causes variations of gene expressions. A passenger is a protein-coding gene or microRNA whose expression is modulated by the driver molecular aberration. Notice our definitions of drivers and passengers specify mechanistic/causal relations of genes but do not require information about clinical phenotypes. We term the conventional definitions of drivers and passengers as phenotypic and the extended definitions as mechanistic. A phenotypic driver is usually also a mechanistic driver, since it may affect clinical phenotypes by altering the regulatory programs in cancer cells. Conversely, a mechanistic driver may not be a phenotypic driver if the altered passenger genes do not pertain to the clinical phenotypes. Similarly, a mechanistic passenger can be a phenotypic driver if the expression change of the gene affects the cancer phenotype.

Comprehensive characterization of diverse molecular aberrations in tumors is a major trend of cancer research in the post-genomic era. Several international consortia and research institutions have launched large-scale projects to catalog the genomic, transcriptomic and epigenetic changes across multiple tumor types. Two noted endeavors are The Cancer Genome Atlas (TCGA) [7] and the International Cancer Genome Consortium (ICGC) [8]. Beyond these coordinated efforts, comprehensive assays on the NCI-60 cell lines have been performed by distinct research groups over the last two decades (e.g., [9-19]).

As large-scale, comprehensive cancer genomic data become more abundant, it is essential to develop computational tools to integrate heterogeneous data in order to acquire a systematic understanding of cancer cells. A rich collection of previous studies attempted to identify the driver mutations responsible for tumorigenesis from cancer genomic data (e.g., $[2,20])$. These studies hypothesized that the driver mutations conferred selective advantages for tumor growth thus occurred in multiple independent instances. Accordingly they aimed for finding recurrent aberrations with high frequencies. Beyond single genes a variety of computational tools have been proposed to examine the abnormal pathway activities by combining the molecular information of their constituent genes, such as the gene set enrichment analysis [21], principal component analysis [22], factor graph models [23], and others (e.g., [24]). Causal and mechanistic relations of molecular aberrations and gene expressions were not addressed in these studies. Some studies tracked the causes of abnormal gene expressions by correlating them with DNA copy numbers, gene mutations, DNA methylations or microRNA expressions (e.g., $[7,25-27,4])$. However, these studies were often restricted to pairwise comparisons between two types of data and lacked a unifying framework to integrate multiple types of data in the same model.
Beyond cancer data analysis many generic computational models of data integration have been proposed. Causal relations were constrained by a variety of properties such as conditional independence of observed variables (e.g., [28,29]), molecular interactions and pathways (e.g., [30]), and data generated from intervention experiments (e.g., [31]). While many of these tools have been applied to cancer data, most of the questions of interest are phenotype-driven (e.g., finding genes responsible for a clinical trait) rather than mechanism-driven (e.g., finding the causal relations connecting driver aberrations and passenger gene expressions).

Akavia et al. inferred mechanistic relations of gene expressions and phenotypes in melanoma [32]. They identified the gene copy number changes that modulated their own expressions and indirectly affected the expressions of their target genes. Curiously, these mechanistic drivers were also phenotypic drivers, as their knock-downs abrogated tumor cell proliferation. Despite the value of connecting mechanistic and phenotypic characterization, this study was restricted to copy number variations and discarded other types of molecular aberrations.

Recently, we proposed a layered modeling framework for integrative analysis of cancer genomic data [33]. The goal was to explain gene expressions with observed molecular aberrations. Associations with molecular aberrations were incrementally included according to levels of uncertainty and mechanistic information. In this work, we extended and modified the layered modeling framework to identify association modules of driver molecular aberrations and passenger gene/mircroRNA expressions from the integrated datasets of NCI-60 cell lines. For each type of molecular aberrations, we found the downstream passengers putatively affected by the drivers and regulators mediating the effects from drivers to passengers. The causal relations between drivers/regulators and passengers were supported by both experimental and in-silico validations. The analysis results justify the utility of association modules for other cancer genomic data.

\section{Results}

\section{Construction of association modules from integrated datasets}

Diverse molecular aberrations can modulate variations of gene expressions in cancer cells. The dependencies between molecular aberrations and gene expressions may be modular, as multiple genes with coherent expression profiles are likely to be affected by common molecular aberrations. The goal of this study is to find the modules of genes or microRNAs whose coherent expression profiles are possibly driven by common molecular aberrations. We define an association module 
as a tuple consisting of three components: (1)observed driver molecular aberrations, (2)passen-ger genes or microRNAs whose expression profiles are associated with driver molecular aberrations, (3)regulators (transcription factors) that mediate the effects between drivers and passengers. We consider the following types of association modules:

1. Cis-acting effects with copy number variations of chromosomal segments. The copy number variation (CNV) of a chromosomal segment is associated with the expressions of its constituent genes or microRNAs.

2. Trans-acting effects with copy number variations of chromosomal segments. A chromosomal segment CNV possesses cis-acting effects with intermediate regulators on the segment, and both segment CNV and regulator expressions are associated with the expressions of genes or microRNAs on other segments.
3. Effects with gene mutations. The mutational state of a gene is associated with the expressions of itself and other genes or microRNAs.

4. Effects with DNA methylations. The coherent DNA methylation state of a collection of genes is negatively associated with the expressions of genes or microRNAs.

5. Regulatory effects with microRNAs. The coherent expressions of a collection of microRNAs are negatively associated with the expressions of a collection of genes.

6. Regulatory effects with transcription factors. The coherent expressions of a collection of transcription factors are associated with the expressions of a collection of other genes.

The association modules are visually summarized in Figure 1 and elaborated in Materials and Methods and Additional file 1, Text S1.

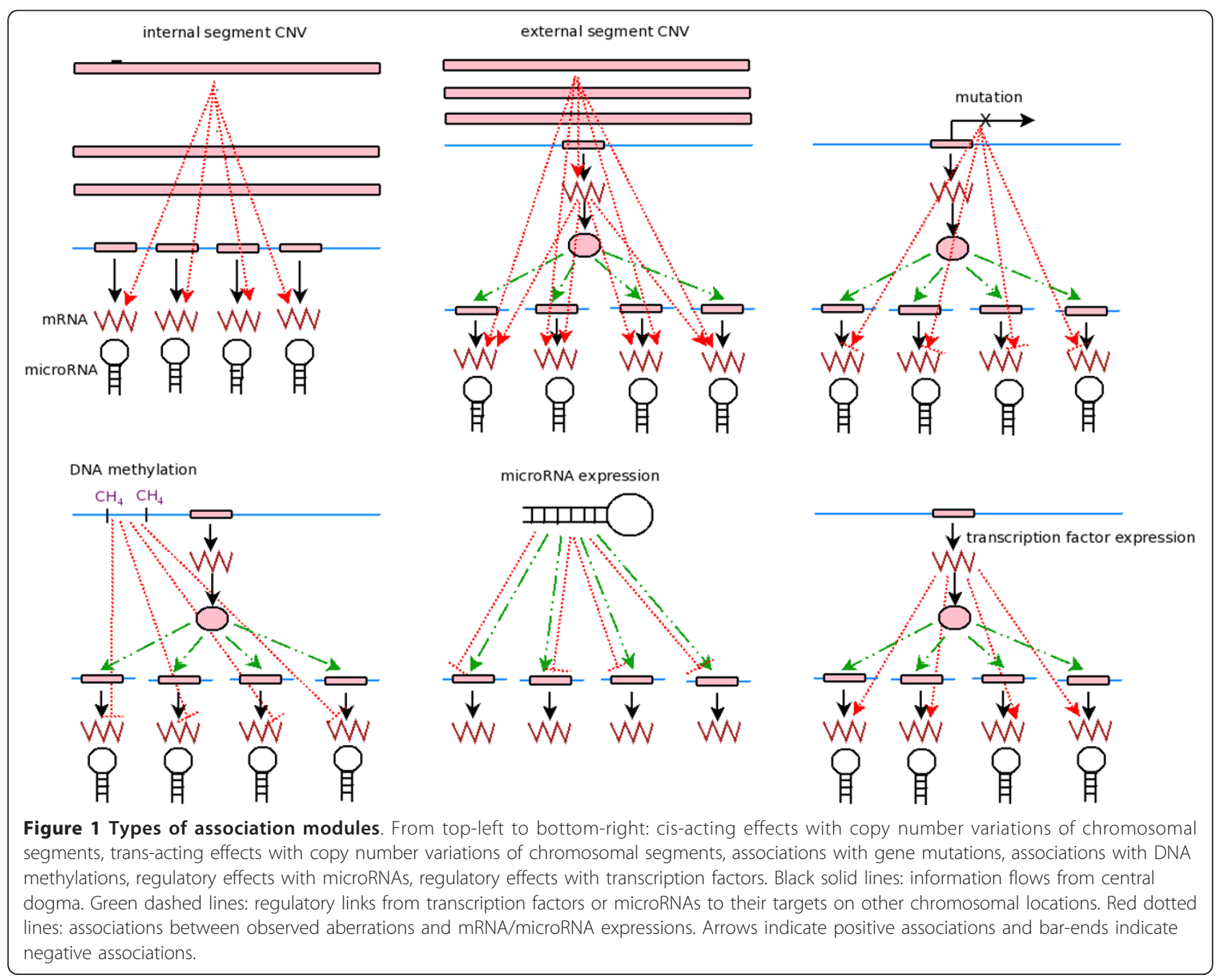


Associations between driver aberrations and passenger expressions are established by a series of hypothesis testing procedures using logistic regression models. In brief, an association between a driver and a passenger is selected only if it cannot be replaced by any other association (with the same passenger) without a significant loss of the explanatory power. Procedures of constructing association modules are described in Materials and Methods and Additional file 1, Text S1.

\section{Diverse molecular aberrations drive mRNA responses in $\mathrm{NCl}-60$ cell lines}

We identified association modules from integrated datasets of NCI-60 cell lines, including mRNA and microRNA expressions, copy number variations, mutations, and DNA methylations (see Materials and Methods for the data sources and processing). 6888 genes possessed coherent expression profiles among three mRNA datasets. $48.16 \%$ (3317 of 6888 ) of the valid mRNA expression profiles were explained by observed molecular aberrations. Intriguingly, each type of association modules in Figure 1 were detected, suggesting diverse molecular mechanisms drive mRNA expressions.

\section{Summary of $M R N A$ association modules}

Table 1 shows the summary information of association modules for mRNA expressions. Associations with segment CNVs dominate the inferred modules. 1699 of 3317 passenger expressions explained by observed aberrations are associated with CNVs on their own segments, and 43 of 84 association modules are cis-acting effects with segment CNVs. Trans-acting effects with segment CNVs comprise 18 association modules and 506 passenger genes. 522 passenger expressions and 14 association modules are positively or negatively associated with mutations of 8 oncogenes and tumor suppressors. 161 passenger expressions and 5 association modules are negatively associated with DNA methylations. Two association modules with microRNA expressions comprise 121 and 120 passenger genes respectively. Moreover, two association modules with transcription factor expressions comprise 338 and 338 passenger genes respectively. The complete list of association modules are reported in Additional file 2, Table $\mathrm{S} 1$, and driver aberrations and passenger mRNA expressions are visualized in Additional file 1, Figures S1, S2, S3, S4, S5, S6, S7, S8, S9, S10, S11, S12 and S13. The complete list of microRNA association modules are reported in Additional file 3, Table S2, and driver aberrations and passenger microRNA expressions are visualized in Additional file 1, Figures S14, S15, S16.

The credibility of associations between multiple variables was assessed by false discovery rates (FDR,
$[34,35])$. We adopted the permutation tests described in [36] as the null model and evaluated two types of FDRs: (1) $\frac{\text { expected \# false positives according to the null model }}{\text { ppositive calls from the date }}$ (2) $\frac{\text { \# false positives in the } 99 \text { percentile of the null model }}{\text { positive calls from the date }}$

Table 2 shows the FDRs for each type of associations and for all associations together. The FDRs for cis and trans acting effects with segment CNVs are substantially smaller than those for other types of associations. This is sensible as the cis-acting effects with segment CNVs are constrained by chromosomal locations of passenger genes, and the trans-acting effects with segment CNVs are constrained by coherent segment CNVs and expressions of their regulators. In contrast, other types of associations possess no additional constraints beyond driver aberrations and passenger expressions, thus are more likely to be spurious. Furthermore, the FDRs calculated from the expected number of false positives are lower than those calculated from the 99 percentile of the null distribution. This is also sensible since the latter gives a much more conservative estimate of the false positive numbers. The overall FDRs calculated by these two methods are 0.235 and 0.326 respectively.

To justify the biological meanings of association modules, we conducted several in-silico validations based on prior literature. First, we extracted putative targets of transcription factors from the TRANSFAC database [37] and those of microRNAs from three databases ([38-40]). For each association module, we then evaluated the enrichment p-values for the putative targets of their regulators (transcription factors or microRNAs) in the passenger genes. Table 3 reports the enrichment $\mathrm{p}$-values for the putative targets of regulators in the association modules. Among the 14 modules whose regulators possess putative target information, 9 of them are significantly enriched ( $\mathrm{p}$-value $<0.05$ ) with putative targets in their passenger genes. All but one modules containing more than 60 passenger genes are enriched with putative targets of their regulators. Only one large module positive associations with APC mutations - is not enriched with the putative targets of the regulator since $\mathrm{APC}$ is not a transcription factor and has no binding motifs.

Second, we incurred a batch search for all pairs of drivers/regulators and passengers of association modules on NCBI PubMed database and checked whether they were co-cited in previous publications. Table 4 summarizes the information of co-cited (driver/regulator, passenger) pairs for each association module. In total, 449 (driver/regulator, passenger) pairs co-occur in the same publications, and 22 of 46 association modules with non-local aberrations have co-cited (driver/regulator, passenger) pairs. To assess the significance of co-citations we randomly sampled passenger 
Table 1 Summary information of association modules for mRNA expressions

\begin{tabular}{|c|c|c|c|c|c|c|c|c|c|c|c|}
\hline index & driver type & drivers & reg & sign & $N$ & index & driver type & drivers & reg & sign & $N$ \\
\hline 1 & intra CNV & seg 30 (chr 6p) & NA & + & 84 & 44 & inter CNV & seg 18 (chr 3p) & ZIC1,MITF & + & 118 \\
\hline 2 & intra CNV & seg $54(\operatorname{chr} 12 q)$ & NA & + & 77 & 45 & inter CNV & seg 31 (chr 6q) & TBP,MYB,HDAC2 & + & 77 \\
\hline 3 & intra CNV & seg 1 (chr 1p) & NA & + & 74 & 46 & inter CNV & seg 84 (chr 22q) & SREBF2,EP300 & + & 49 \\
\hline 4 & intra CNV & seg 57 (chr 14q) & NA & + & 62 & 47 & inter CNV & seg 30 (chr 6p) & E2F3,NFYA,SRF & + & 31 \\
\hline 5 & intra CNV & seg 69 (chr 17q) & NA & + & 62 & 48 & inter CNV & seg 42 (chr 10q) & TCF7L2 & + & 30 \\
\hline 6 & intra CNV & seg 11 (chr 2p) & NA & + & 58 & 49 & inter CNV & seg 55 (chr 12q) & $\mathrm{NR} 2 \mathrm{C} 1, \mathrm{NFYB}$ & + & 22 \\
\hline 7 & intra CNV & seg 42 (chr 10q) & NA & + & 55 & 50 & inter CNV & seg 19 (chr 3q) & ZNF148 & + & 22 \\
\hline 8 & intra CNV & seg 3 (chr 1p) & NA & + & 53 & 51 & inter CNV & seg 63 (chr 16pq) & NFATC3,RBL2 & + & 21 \\
\hline 9 & intra CNV & seg 37 (chr 8q) & NA & + & 53 & 52 & inter CNV & seg 69 (chr 17q) & SPOP,NME2,POLG2 & + & 20 \\
\hline 10 & intra CNV & seg 59 (chr 14q) & NA & + & 53 & 53 & inter CNV & seg 42 (chr 10q) & NFKB2 & + & 19 \\
\hline 11 & intra CNV & seg 16 (chr 3p) & NA & + & 49 & 54 & inter CNV & seg 16 (chr 3p) & UBP1 & + & 18 \\
\hline 12 & intra CNV & seg 63 (chr 16pq) & NA & + & 49 & 55 & inter CNV & seg 83 (chr 22q) & EWSR1 & + & 17 \\
\hline 13 & intra CNV & seg 33 (chr 7q) & NA & + & 47 & 56 & inter CNV & seg 57 (chr 14q) & APEX1 & + & 16 \\
\hline 14 & intra CNV & seg 84 (chr 22q) & NA & + & 47 & 57 & inter CNV & seg 16 (chr 3p) & RPA2,EIF2C1 & + & 15 \\
\hline 15 & intra CNV & seg 55 (chr 12q) & NA & + & 44 & 58 & inter CNV & seg 19 (chr 3q) & CNBP & + & 15 \\
\hline 16 & intra CNV & seg 51 (chr 11q) & NA & + & 43 & 59 & inter CNV & seg 51 (chr 11q) & RICS & + & 14 \\
\hline 17 & intra CNV & seg 2 (chr 1p) & NA & + & 42 & 60 & inter CNV & seg 56 (chr 13q) & GTF3A & + & 13 \\
\hline 18 & intra CNV & seg 73 (chr 19p) & NA & + & 42 & 61 & inter CNV & seg 4 (chr 1q) & RUNX3 & + & 10 \\
\hline 19 & intra CNV & seg 50 (chr 11q) & NA & + & 40 & 62 & mutation & $\mathrm{APC}$ & NA & + & 136 \\
\hline 20 & intra CNV & seg 68 (chr 17q) & NA & + & 39 & 63 & mutation & APC & NA & - & 56 \\
\hline 21 & intra CNV & seg 65 (chr 17p) & NA & + & 37 & 64 & mutation & PIK3CA & NA & + & 50 \\
\hline 22 & intra CNV & seg 32 (chr 7p) & NA & + & 36 & 65 & mutation & CDKN2A & NA & - & 43 \\
\hline 23 & intra CNV & seg 35 (chr 7q) & NA & + & 35 & 66 & mutation & TP53 & NA & - & 35 \\
\hline 24 & intra CNV & seg 31 (chr 6q) & NA & + & 33 & 67 & mutation & BRAF & NA & - & 34 \\
\hline 25 & intra CNV & seg 19 (chr 3q) & NA & + & 32 & 68 & mutation & TP53 & NA & + & 33 \\
\hline 26 & intra CNV & seg 18 (chr 3p) & NA & + & 30 & 69 & mutation & CDKN2A & NA & + & 32 \\
\hline 27 & intra CNV & seg 9 (chr 1q) & NA & + & 29 & 70 & mutation & KRAS & NA & + & 25 \\
\hline 28 & intra CNV & seg 22 (chr 4p) & NA & + & 29 & 71 & mutation & PTEN & NA & + & 20 \\
\hline 29 & intra CNV & seg 62 (chr 15q) & NA & + & 29 & 72 & mutation & KRAS & NA & & 16 \\
\hline 30 & intra CNV & seg 41 (chr 9q) & NA & + & 28 & 73 & mutation & BRAF & NA & + & 16 \\
\hline 31 & intra CNV & seg 49 (chr 11q) & NA & + & 28 & 74 & mutation & PIK3CA & NA & - & 13 \\
\hline 32 & intra CNV & seg 56 (chr 13q) & NA & + & 28 & 75 & mutation & PTEN & NA & - & 13 \\
\hline 33 & intra CNV & seg 60 (chr 15q) & NA & + & 28 & 76 & methylation & PAX8 & NA & - & 61 \\
\hline 34 & intra CNV & seg 44 (chr 11p) & NA & + & 27 & 77 & methylation & $\mathrm{BCR}$ & NA & - & 58 \\
\hline 35 & intra CNV & seg 61 (chr 15q) & NA & + & 26 & 78 & methylation & HOXC13 & NA & - & 19 \\
\hline 36 & intra CNV & seg 80 (chr 21q) & NA & + & 24 & 79 & methylation & CCND1,PPARG & NA & - & 13 \\
\hline 37 & intra CNV & seg 36 (chr 8p) & NA & + & 23 & 80 & methylation & COL5A1 & NA & - & 10 \\
\hline 38 & intra CNV & seg 27 (chr 5q) & NA & + & 22 & 81 & microRNA & mir group 1 & NA & - & 121 \\
\hline 39 & intra CNV & seg 39 (chr 9p) & NA & + & 22 & 82 & microRNA & mir group 2 & NA & - & 120 \\
\hline 40 & intra CNV & seg 12 (chr 2q) & NA & + & 20 & 83 & TF & TF group 1 & NA & + & 338 \\
\hline 41 & intra CNV & seg 74 (chr 19q) & NA & + & 20 & 84 & TF & TF group 2 & NA & + & 338 \\
\hline 42 & intra CNV & seg 83 (chr 22q) & NA & + & 20 & & & & & & \\
\hline 43 & intra CNV & seg 85 (chr 23pq) & NA & + & 20 & & & & & & \\
\hline
\end{tabular}

Index: module index. Reg: intermediate regulators. Sign: the functional direction (activation or repression) from drivers to passengers. $N$ : number of passenger genes. Intra CNV: intra-segment CNV. Inter CNV: inter-segment CNV. TF: transcription factor expressions. The locations of segments are reported in Supplementary Table S5. Mir group 1 consists of mir-92,mir-96,mir-106a,mir 20b,mir-17,mir-19b,mir-32,mir-135,mir-25,mir-106b,mir-93,mir-106,mir-18,mir-20. Mir group 2 consists of mir-24,mir-99b,mir-27b,mir-21,mir-125a,mir-23b,mir-27a,mir-23a. TF group 1 consists of SMAD3,FOXD1,PLAU,BDNF,FOSL2,FOSL1,RBMS1. TF group 2 consists of ERG,ELK4,NFATC1,RFX3,POU5F1,ZNF350. 
Table 2 False discovery rates of mRNA and microRNA association modules

\begin{tabular}{|c|c|c|}
\hline \multicolumn{3}{|l|}{ mRNA modules: } \\
\hline FDR evaluation & driver type & FDR \\
\hline mean & intra CNV & 0.0259 \\
\hline mean & inter CNV & 0.0035 \\
\hline mean & mutation & 0.3467 \\
\hline mean & methylation & 0.4334 \\
\hline mean & microRNA & 0.2248 \\
\hline mean & $\mathrm{TF}$ & 0.2197 \\
\hline mean & all & 0.2353 \\
\hline $99 \%$ & intra CNV & 0.0855 \\
\hline $99 \%$ & inter CNV & 0.0899 \\
\hline $99 \%$ & mutation & 0.4949 \\
\hline $99 \%$ & methylation & 0.6322 \\
\hline $99 \%$ & microRNA & 0.3109 \\
\hline $99 \%$ & $\mathrm{TF}$ & 0.2815 \\
\hline $99 \%$ & all & 0.3262 \\
\hline \multicolumn{3}{|c|}{ microRNA modules: } \\
\hline FDR evaluation & driver type & FDR \\
\hline mean & intra CNV & NA \\
\hline mean & inter CNV & 0.0021 \\
\hline mean & mutation & 0.2535 \\
\hline mean & methylation & 0.4403 \\
\hline mean & microRNA & NA \\
\hline mean & TF & 0.2857 \\
\hline mean & all & 0.2924 \\
\hline $99 \%$ & intra CNV & NA \\
\hline $99 \%$ & inter CNV & 0.0455 \\
\hline $99 \%$ & mutation & 0.3645 \\
\hline $99 \%$ & methylation & 0.6379 \\
\hline $99 \%$ & microRNA & NA \\
\hline $99 \%$ & $\mathrm{TF}$ & 0.3600 \\
\hline $99 \%$ & all & 0.3943 \\
\hline
\end{tabular}

Mean: using the expected number of false positives to evaluate FDR. 99\%: using the 99 percentile of the number of false positives to evaluate FDR. Intra CNV: intra-segment CNV. Inter CNV: inter-segment CNV. TF: transcription factor expressions. All: the FDR over all associations.

genes of each module and counted the maximum number of co-cited pairs over 10 random trials. Among the 23 association modules possessing co-cited pairs, 7 of them have more than 1.4 fold of the number of co-cited pairs compared to random samples (Table 4). The complete co-cited (driver/regulator, passenger) pairs among all association modules are reported in Additional file 4, Table S3.

Third, for the passenger genes in each association module we evaluated the enrichment $\mathrm{p}$-values for functional categories [41] and curated pathways ([42-44]). Table 5 reports representative GO categories and pathways. 38 of the 84 association modules have at least one enriched GO category/pathway with p-value $\leq 0.001$.
Table 3 Enrichment of driver/regulator binding motifs on passenger promoters of mRNA association modules

\begin{tabular}{|c|c|c|c|c|c|}
\hline index & reg & $N_{1}$ & $N_{2}$ & $N_{3}$ & $p$-value \\
\hline 44 & MITF & 118 & 2023 & 23 & 0.0464 \\
\hline 45 & $\mathrm{TBP}$ & 77 & 8009 & 56 & $5.3704 \times 10^{-4}$ \\
\hline 45 & MYB & 77 & 10684 & 65 & 0.0076 \\
\hline 46 & SREBF2 & 49 & 9755 & 36 & 0.1581 \\
\hline 47 & E2F3 & 31 & 8123 & 21 & 0.0989 \\
\hline 47 & NFYA & 31 & 2170 & 3 & 0.8513 \\
\hline 47 & SRF & 31 & 75 & 0 & 1.0 \\
\hline 51 & NFATC3 & 22 & 2170 & 5 & 0.2097 \\
\hline 53 & NFKB2 & 19 & 531 & 0 & 1.0 \\
\hline 66 & TP53 & 35 & 3576 & 16 & 0.0041 \\
\hline 76 & PAX8 & 61 & 5376 & 34 & 0.0014 \\
\hline 78 & HOXC13 & 19 & 5421 & 12 & 0.0164 \\
\hline 79 & PPARG & 13 & 20 & 0 & 1.0 \\
\hline 81 & mir-96 & 121 & 636 & 11 & 0.0147 \\
\hline 81 & mir-106a & 121 & 319 & 6 & 0.0463 \\
\hline 81 & mir-17 & 121 & 830 & 12 & 0.0379 \\
\hline 81 & mir-93 & 121 & 832 & 12 & 0.0385 \\
\hline 81 & mir-106 & 121 & 718 & 11 & 0.0323 \\
\hline 82 & mir-21 & 120 & 291 & 7 & 0.0093 \\
\hline 83 & SMAD3 & 338 & 10025 & 271 & $1.058 \times 10^{-7}$ \\
\hline 84 & ERG & 338 & 2231 & 67 & 0.0093 \\
\hline 84 & ELK4 & 338 & 2803 & 76 & 0.0518 \\
\hline 84 & NFATC1 & 338 & 10187 & 265 & $3.1517 \times 10^{-5}$ \\
\hline 84 & RFX3 & 338 & 2751 & 75 & 0.0481 \\
\hline 84 & POU5F & 338 & 379 & 18 & 0.0027 \\
\hline
\end{tabular}

Index: module index. Reg: the transcription factor or microRNA with putative targets. $N_{1}$ : number of passenger genes in a module. $N_{2}$ : total number of putative targets in human genes. $N_{3}$ : number of passenger genes containing the transcription factor binding motif. p-value: hyper-geometric $p$-value of enrichment. For modules with microRNAs as drivers, $N_{2}$ : number of putative targets of a microRNA. $N_{3}$ : number of passenger genes belonging to the putative targets of the microRNA.

The complete list of enriched GO categories and pathways are reported in Additional file 5, Table S4.

Genes of cis-acting effects with segment CNVs exhibit a clustering tendency

We partitioned the CGH data into 86 coherent segments (Additional file 1, Text S1) and examined positive associations between segment CNVs and the mRNA expressions of their constituent genes. The cis-acting effects with segment CNVs constitute a substantial portion of the associations with mRNA expressions: 1699 of 6888 mRNA expressions can be explained by the local segment CNVs. These associations are grouped by segments into 43 modules. The members of association modules for local segment CNVs are reported in Additional file 2, Table S1, and information about partitioned segments is reported in Additional file 6, Table S5.

The passenger genes associated with local segment $\mathrm{CNVs}$ are not evenly distributed on chromosomes. Figure 2 marks the locations of these passenger genes (blue 
Table 4 Summary of co-citations in mRNA and microRNA association modules

\begin{tabular}{|c|c|c|c|c|}
\hline \multicolumn{5}{|c|}{ mRNA modules } \\
\hline index & drivers/regulators & $N_{1}$ & $N_{2}$ & $N_{3}$ \\
\hline 44 & ZIC1,MITF seg 18 CNV (+) & 118 & 14 & 6 \\
\hline 45 & TBP,MYB,HDAC2 seg 31 CNV (+) & 77 & 28 & 20 \\
\hline 46 & SREBF2,EP300 seg 84 CNV (+) & 49 & 3 & 2 \\
\hline 47 & E2F3,NFYA,SRF seg 30 CNV (+) & 31 & 5 & 4 \\
\hline 48 & TCF7L2 seg 42 CNV $(+)$ & 30 & 1 & 3 \\
\hline 54 & UBP1 seg 16 CNV (+) & 18 & 1 & 0 \\
\hline 62 & APC mutation $(+)$ & 136 & 17 & 10 \\
\hline 63 & APC mutation (-) & 56 & 3 & 6 \\
\hline 65 & CDNK2A mutation (-) & 43 & 3 & 6 \\
\hline 66 & TP53 mutation (-) & 35 & 11 & 3 \\
\hline 67 & BRAF mutation (-) & 34 & 3 & 3 \\
\hline 68 & TP53 mutation (+) & 33 & 4 & 6 \\
\hline 69 & CDKN2A mutation $(+)$ & 32 & 6 & 3 \\
\hline 71 & PTEN mutation $(+)$ & 20 & 1 & 2 \\
\hline 72 & KRAS mutation (-) & 16 & 1 & 1 \\
\hline 73 & BRAF mutation $(+)$ & 16 & 3 & 1 \\
\hline 75 & PTEN mutation (-) & 13 & 2 & 4 \\
\hline 76 & PAX8 methylation (-) & 67 & 1 & 4 \\
\hline 77 & BCR methylation (-) & 58 & 5 & 10 \\
\hline 79 & CCND1,PPARG methylation (-) & 13 & 4 & 7 \\
\hline 80 & COL5A1 methylation (-) & 10 & 1 & 1 \\
\hline 83 & TF group $1(+)$ & 338 & 236 & 48 \\
\hline \multicolumn{5}{|c|}{ microRNA modules } \\
\hline index & drivers/regulators & $\mathrm{N}_{1}$ & $\mathrm{~N}_{2}$ & $\mathrm{~N}_{3}$ \\
\hline 4 & ZIC1,MITF seg 18 CNV (-) & 19 & 3 & 5 \\
\hline 5 & DNMT1 seg 15 CNV (+) & 15 & 1 & 2 \\
\hline 6 & SRF seg 30 CNV (+) & 13 & 1 & 1 \\
\hline 7 & MAP2K4 seg 66 CNV (-) & 13 & 2 & 2 \\
\hline 10 & TP53 mutation $(+)$ & 17 & 3 & 3 \\
\hline 12 & APC mutation (+) & 10 & 1 & 1 \\
\hline 14 & BCR methylation (-) & 12 & 1 & 4 \\
\hline 15 & SMAD3,BDNF (+) & 28 & 8 & 10 \\
\hline 20 & $\operatorname{HIF1A}(+)$ & 14 & 1 & 2 \\
\hline
\end{tabular}

Index: module index. $N_{1}$ : number of passengers in the module. $N_{2}$ : number of co-cited driver/regulator-passenger pairs in the module. $N_{3}$ : max number of co-cited driver/regulator-passengers over 10 random modules of the same size. TF group 1 consists of SMAD3,FOXD1,PLAU,BDNF,FOSL2,FOSL1,RBMS. TF group 2 consists of ERG,ELK4,NFATC1,POU5F1,ZNF350.

lines) on each chromosome. The passenger genes associated with local segment CNVs exhibit a clustering tendency. To rule out the possibility that the clustering patterns arise from the distributions of all protein-coding genes on chromosomes, we also marked the locations of passenger genes associated with non-local segment CNVs (trans-acting effects) in Figure 2 (red lines). Despite the presence of dense clusters, the passenger genes with trans-acting effects are more scattered on chromosomes. We evaluated the entropies (dispersions) of gene densities for cis and trans-acting genes on each chromosome (Figure 2). The trans-acting genes have higher entropies than the cis-acting genes in the majority of the chromosomes, confirming the visual observation about their differences.

\section{Trans-acting effects with segment CNVs are mediated by} transcription factors

506 mRNA expressions are associated with non-local segment CNVs. One possible mechanism to establish these trans-acting associations is through intermediate regulators on segments: segment $\mathrm{CNVs}$ have cis-acting effects on the expressions of their constituent regulators, which in turn modulate the expressions of downstream targets on other loci. We identified 18 association modules with intermediate regulators and reported them in Table 1 . Module 44 has segment 18 (chromosome 3p) as the driver, MITF and ZIC1 as intermediate regulators, and 118 passengers. The driver $\mathrm{CNV}$ as well as regulator and passenger mRNAs all exhibit melanoma-specific elevation in NCI-60 cell lines (Figure 3). MITF encodes a transcription factor that regulates the differentiation and development of melanocytes and pigment cell-specific transcription of the melanogenesis enzyme genes. ZIC1 encodes a zinc finger transcription factor involved in brain development. Multiple lines of evidence support the associations with MITF in this module. First, amplification of the chromosome $3 p$ region covering MITF is considered as a typical driver mutation in melanoma [26]. Second, the passenger genes in this module are enriched with experimentally validated MITF targets from [45] (14 of 118 genes, hyper-geometric p-value $\leq$ $\left.1.036 \times 10^{-13}\right)$, and with genes belonging to the GO category of melanosome ( 4 of 7 genes in the GO category, hyper-geometric $\mathrm{p}$-value $\leq 1.3 \times 10^{-7}$ ). Third, promoters of passenger genes are also enriched with MITF-binding motifs (Table 3, 23 of 118 passenger genes containing the motif, hyper-geometric p-value $\leq$ 0.0464). Fourth, MITF and 12 of the 118 passenger genes are co-cited in previous publications (Table 4).

Module 45 has segment 31 (chromosome 6q) as the driver, MYB, TBP and HDAC2 as intermediate regulators, and 77 passengers. The driver $\mathrm{CNV}$ as well as regulator and passenger mRNAs all exhibit leukemiaspecific elevation (Figure 3). MYB (c-myb) is a transcription factor involved in cell cycle progression, cell proliferation and differentiation in hematopoiesis. Amplifications of this oncogene cause its abnormal expressions in leukemia and other solid tumors [46,47]. TBP is a TATA-binding protein belonging to the general transcription apparatus. HDAC2 is a histone deacetylase that modifies chromatin structures and represses transcription. Associations of MYB and TBP with the passenger genes are supported by enrichment of their binding motifs on promoters (Table 3, 65 of 77 genes 
Table 5 Representative enriched GO categories and pathways in mRNA association modules

\begin{tabular}{|c|c|c|c|c|}
\hline index & drivers/regulators & $N_{1}$ & function & $p$-value \\
\hline 44 & ZIC1,MITF seg 18 CNV (+) & 118 & melanosome & $1.300 \times 10^{-7}$ \\
\hline 45 & TBP,MYB,HDAC2 seg 31 CNV (+) & 77 & RNA polymerase II regulation & $1.35 \times 10^{-5}$ \\
\hline 45 & TBP,MYB,HDAC2 seg 31 CNV (+) & 77 & condensed chromosome & $7.928 \times 10^{-5}$ \\
\hline 46 & SREBF2,EP300 seg 84 CNV (+) & 49 & cholesterol biosynthesis & $3.781 \times 10^{-4}$ \\
\hline 47 & E2F3,NFYA,SRF seg 30 CNV (+) & 31 & ceramide signaling pathway & $4.302 \times 10^{-5}$ \\
\hline 47 & E2F3,NFYA,SRF seg 30 CNV (+) & 31 & NFKB signaling pathway & $8.632 \times 10^{-4}$ \\
\hline 48 & TCF7L2 seg 42 CNV (+) & 30 & multi-drug resistance factors & $5.884 \times 10^{-5}$ \\
\hline 48 & TCF7L2 seg 42 CNV (+) & 30 & positive regulation of epithelial cell proliferation & $1.096 \times 10^{-4}$ \\
\hline 49 & NR2C1,NFYB seg 49 CNV (+) & 22 & mRNA translation & $2.314 \times 10^{-4}$ \\
\hline 54 & UBP1 seg 16 CNV (+) & 18 & RNA Pol II phosphorylation & $4.429 \times 10^{-4}$ \\
\hline 56 & APEX1 seg $57(+)$ & 16 & cleavage of growing transcript & $7.872 \times 10^{-4}$ \\
\hline 57 & RPA2,EIF2C1 seg 16 CNV (+) & 15 & mRNA splicing & $1.945 \times 10^{-6}$ \\
\hline 57 & RPA2,EIF2C1 seg 16 CNV (+) & 15 & mRNA Pol II transcription initiation & $7.260 \times 10^{-4}$ \\
\hline 59 & RICS seg 51 CNV (+) & 14 & regulation of apoptosis & $1.397 \times 10^{-4}$ \\
\hline 62 & APC mutation $(+)$ & 136 & liver development & $4.932 \times 10^{-4}$ \\
\hline 66 & TP53 mutation (-) & 35 & caspase activation & $1.101 \times 10^{-3}$ \\
\hline 66 & TP53 mutation (-) & 35 & P53 signaling pathway & $4.138 \times 10^{-4}$ \\
\hline 68 & TP53 mutation (+) & 33 & cell cycle & $9.787 \times 10^{-4}$ \\
\hline 69 & CDKN2A mutation (+) & 32 & cell motility & $8.510 \times 10^{-4}$ \\
\hline 70 & KRAS mutation $(+)$ & 25 & DNA replication & $2.689 \times 10^{-3}$ \\
\hline 71 & PTEN mutation (+) & 20 & JNK cascade & $2.904 \times 10^{-4}$ \\
\hline 75 & PTEN mutation (-) & 13 & positive regulation of $\mathrm{I}-\kappa \mathrm{B}$ kinase/NF- $\kappa \mathrm{B}$ cascade & $1.745 \times 10^{-3}$ \\
\hline 79 & CCND1,PPARG methylation (-) & 13 & negative regulation of cell proliferation & $6.074 \times 10^{-3}$ \\
\hline 81 & mir group $1(-)$ & 121 & transport vesicle & $3.297 \times 10^{-4}$ \\
\hline 82 & mir group $2(-)$ & 121 & translation & $3.694 \times 10^{-4}$ \\
\hline 83 & TF group $1(+)$ & 338 & integrin signaling pathway & $4.664 \times 10^{-8}$ \\
\hline 83 & TF group $1(+)$ & 338 & TGF $\beta$ signaling pathway & $9.555 \times 10^{-4}$ \\
\hline 84 & TF group $2(+)$ & 338 & T cell differentiation & $1.128 \times 10^{-4}$ \\
\hline
\end{tabular}

Index: module index. $N_{1}$ : number of passengers in the module. Function: enriched GO category or pathway. $p$-value: hyper-geometric $p$-value for enrichment. Mir group 1 consists of mir-92,mir-96,mir-106a,mir 20b,mir-17,mir-19b,mir-32,mir-135,mir-25,mir-106b,mir-93,mir-106,mir-18,mir-20. Mir group 2 consists of mir-24,mir99b,mir-27b,mir-21,mir-125a,mir-23b,mir-27a,mir-23a. TF group 1 consists of SMAD3,FOXD1,PLAU,BDNF,FOSL2,FOSL1,RBMS. TF group 2 consists of ERG,ELK4, NFATC1,POU5F1,ZNF350.

for MYB motifs, hyper-geometric p-value $\leq 0.0076,56$ of 77 genes for TBP motifs, hyper-geometric p-value $\leq$ $5.370 \times 10^{-4}$ ) and co-citations of regulators and passengers in literature (Additional file 4, Table S3, 14 passenger genes are co-cited with $\mathrm{MYB}$, and 8 passenger genes are co-cited with TBP). Intriguingly, the passenger genes in this module are highly enriched with the GO categories or pathways involved in generic DNA and RNA synthesis such as RNA Pol II regulation and chromatin remodeling (Table 5). The elevated activities of DNA and RNA synthesis probably reflect high division rates of leukemia: leukemia has the second lowest (next to colon cancers) average doubling time among the NCI60 cell lines [48].

Associations do not necessarily imply causality as multiple types of causal structures may yield the same statistical associations [29]. We confirmed the causal relations of MYB and its associated genes by intervention experiments. We selected 11 putative target genes whose
mRNAs were associated with segment $31 \mathrm{CNV}$ and MYB expressions in multiple datasets (see Materials and Methods and Additional file 1, Text S1). As a control we selected 6 additional genes with high expression levels across all 60 cell lines. The K562 leukemia cell line was treated with c-myb siRNA, and the expression responses of putative MYB targets and control genes were measured by qPCR. Expressions of 6 of 11 putative MYB targets were down-regulated with the t-test pvalue $\leq 0.05$ under the $c-$ myb siRNA treatment: CTCF, KHDRBS1, NFATC3, ORC1L, PAICS, and ZNF131, and RBMX was significantly up-regulated (Figure 4, green bars; Additional file 7, Table S6). In contrast, the control genes were not differentially expressed under the c-myb siRNA treatment (Figure 4, blue bars; Additional file 7, Table S6). The primers and the probe used for detecting each gene are listed in Additional file 8, Table S7.

Module 46 has segment 84 (chromosome 22q) as the driver, SREBF2 and EP300 as intermediate regulators, 


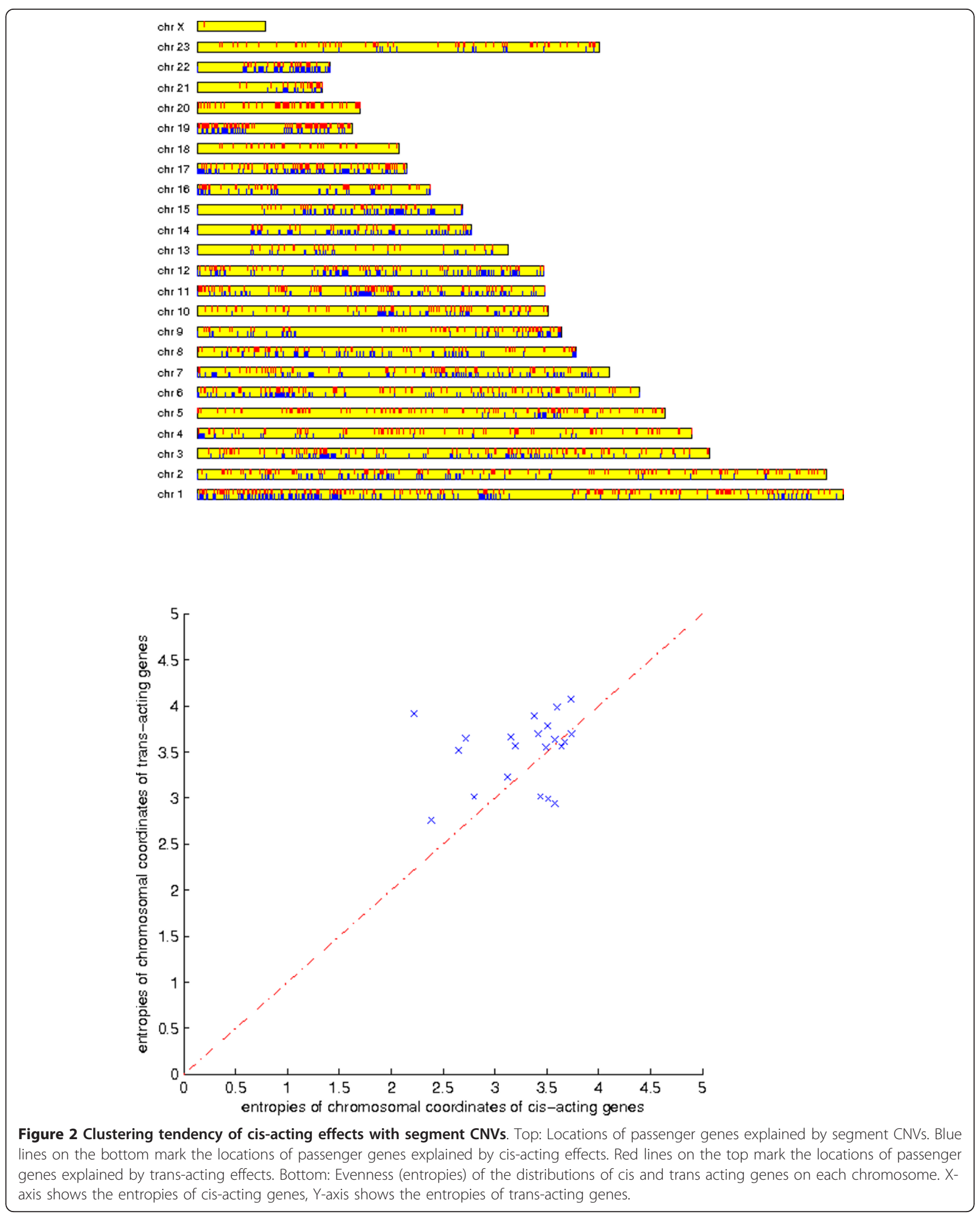




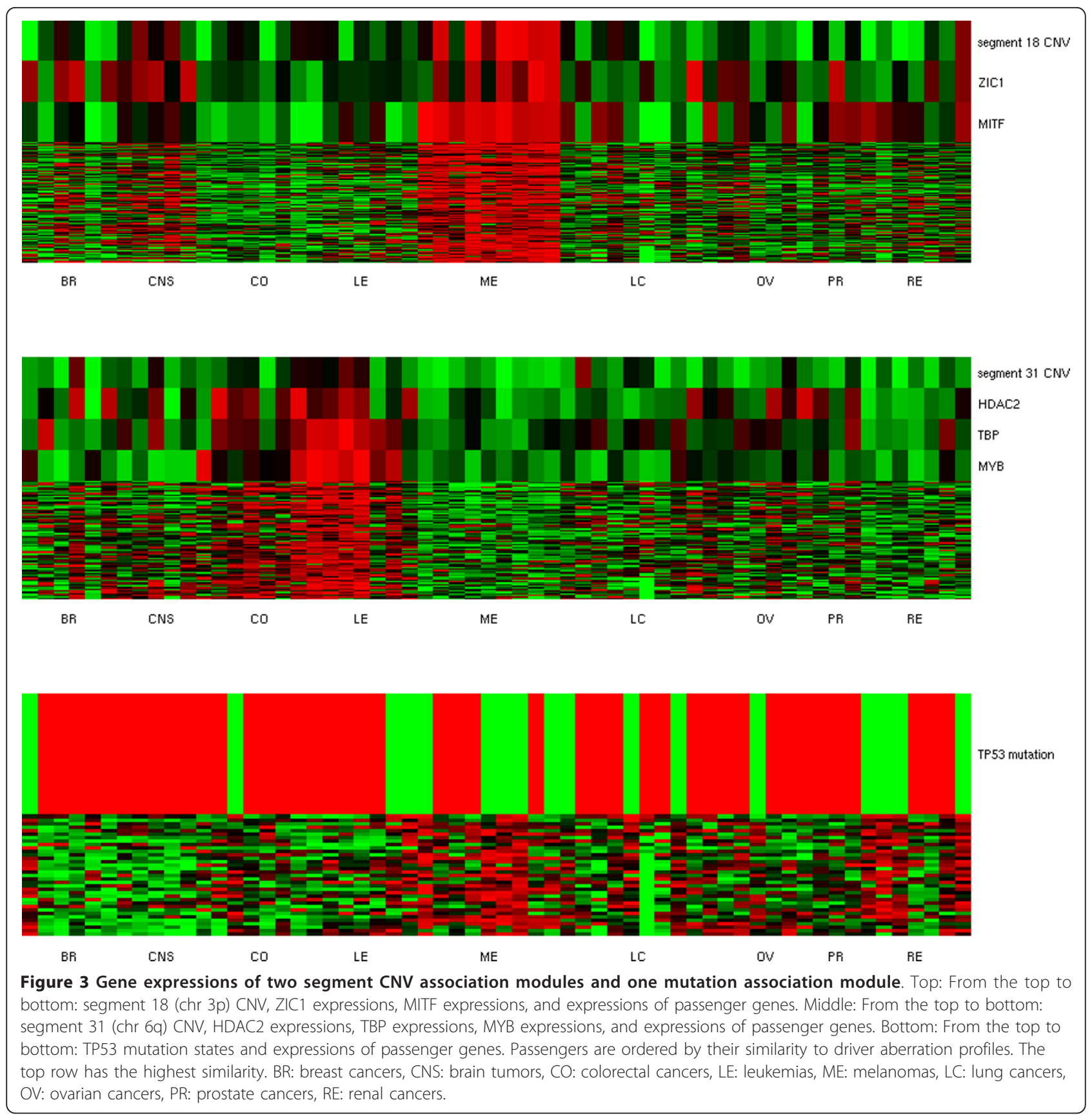

and 49 passengers. SREBF2 encodes a sterol regulatory element binding transcription factor. The SREBF2-binding motif is not enriched on the passenger promoters (hyper-geometric p-value $\leq 0.1581$, Table 3 ). However, two passenger genes are involved in cholesterol biosynthesis (FDFT1 and HMGCR), and the enrichment is significant ( $\mathrm{p}$-value $\leq 3.781 \times 10^{-4}$, Table 5$)$.

Module 47 has segment 30 (chromosome 6p) as the driver, E2F3, NFYA and SRF as intermediate regulators, and 31 passengers. The passenger genes are moderately enriched with E2F3-binding motifs (Table 3, 21 of 31 genes, $\mathrm{p}$-value $\leq 0.0989$ ), and five (regulator, passenger) pairs are co-cited in the same studies (Table 4). SRF (serum response factor) is known to regulate RAF1 (serine/threonine-protein kinase), a member of passenger genes [49]. Moreover, SRF and RAF1 co-participate in multiple signaling pathways including IGF1, PDGF, and MAPK pathways (Additional file 5, Table S4). SRF is also associated with two other passenger genes (RELA and SMAD4) in previous studies (Additional file 4, Table S3). 

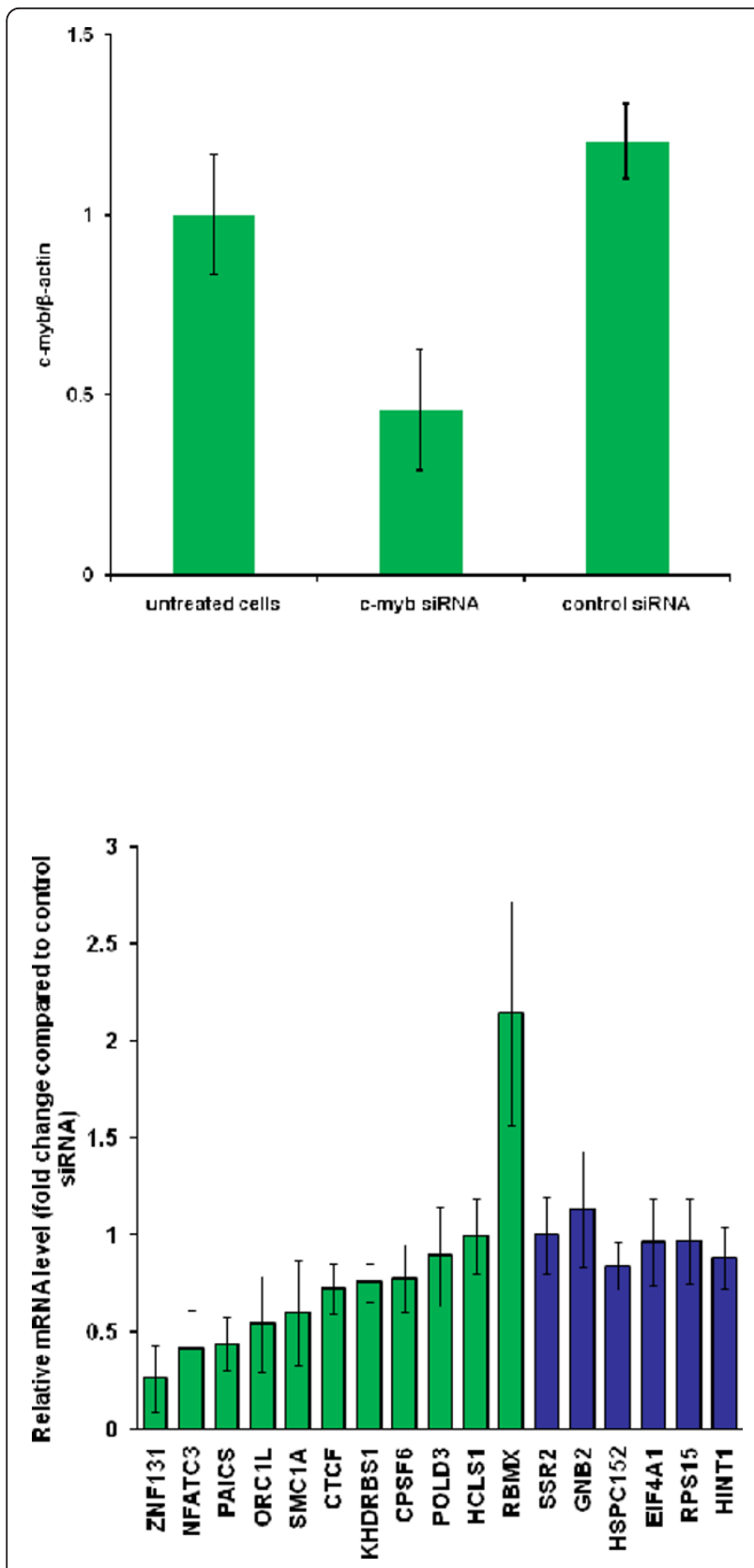

Figure 4 Validation of putative MYB targets with siRNA treatments. Top: qRT-PCR measurements of c-myb mRNA levels in the K562 leukemia cell line without treatment (left), treated with the siRNA against c-myb (middle), and treated with the siRNA against GFP (right). Bottom: Relative mRNA levels of selected genes under the c-myb siRNA treatment with respect to the qRT-PCR measurements under the control siRNA treatment. Green bars: expression responses of putative MYB targets. Blue bars: expression responses of control genes.

Associations with aberrations on individual genes or microRNAs reveal the causal relations of their targets Associations with aberrations on individual genes or microRNAs provide direct causal explanations for gene expressions. 894 mRNA expressions are associated with aberrations on individual genes or microRNAs - mutations, DNA methylations of genes and microRNA expressions. They are grouped into 21 modules and are reported in Table 1 . The causal relations between drivers and passengers are supported by previous studies in 20 association modules.

Positive and negative associations with gene mutations comprise 12 mRNA expression modules and 522 passenger genes. APC encodes a tumor suppressor protein that acts as an antagonist of the Wnt signaling pathway and is involved in other processes such as cell migration and adhesion. APC is mutated exclusively in colon cancer cell lines. APC mutations are positively associated with 136 passenger genes and negatively associated with 56 passenger genes (Additional file 1, Figure S9). More previous studies support the positive associations with APC mutations: 21 passenger genes are co-cited with APC in previous publications. Some of these passenger genes are members or downstream targets of the Wnt signaling pathway: BMP4, CD9, CDH1, GPX2, HDAC1, PPARG, and SOX9 (Additional file 4, Table S3). Mutations of APC activate the Wnt pathway thus up-regulate its target genes. Other passenger genes are associated with APC aberrations in selected tissue or disease samples: DDR1, DLG3, ETS2, HOXA10, ITGB4, MEST, PLA2G4B, SIGIRR, and SPINT2 (Additional file 4, Table S3). In contrast, only 4 negative associations with APC are supported by prior publications. APC and PSMD2 are in the pathway of $\beta$-catenin degradation (Additional file 5, Table S4). APC and CMTM3, EXO1 and HLTF are reported to be hyper-methylated in selected colon cancer samples (Additional file 4, Table S3).

CDKN2A (TP16) encodes a tumor suppressor that inhibits cyclin-dependent kinase 4 (CDK4) in cell cycle control. It possesses loss-of-function mutations (mostly frameshift insertions/deletions) in 33 samples. CDKN2A mutations are negatively associated with 43 passenger genes and positively associated with 32 passenger genes (Additional file 1, Figure S10). Three negative associations are supported by previous studies: ERBB3, FOS and HSD17B4 (Additional file 4, Table S3). Six positive associations are supported by previous studies: CAV1, LIMK1, MSN, THBD, TRDMT1, and YWHAG (Additional file 4, Table S3). Moreover, the positively associated genes are enriched with the GO category of cell motility ( $\mathrm{p}$-value $\leq 8.51 \times 10^{-4}$ ) and signaling pathways of cell motility $\left(\mathrm{p}\right.$-value $\left.\leq 2.28 \times 10^{-3}\right)$ and integrin $(\mathrm{p}$ value $\left.\leq 2.42 \times 10^{-3}\right)$

TP53 encodes a master regulator for apoptosis, cell cycle control and senescence in response to stress conditions. TP53 mutations are negatively associated with 35 passenger genes and positively associated with 33 passenger genes (Additional file 1, Figure S9). Negative 
associations are highly enriched with known TP53 targets from multiple lines of evidence. First, the passenger genes are enriched with a list of 45 known TP53responsive genes confirmed by ChIP-Seq assays ([50], 7 of 35 genes, hyper-geometric p-value $\leq 7.6608 \times 10^{-12}$ ). Second, promoters of the passenger genes are enriched with TP53-binding motifs (16 of 35 genes, hyper-geometric p-value $\leq 0.0041$, Table 3 ). Third, 11 of the 35 passenger genes are co-cited with TP53 in previous publications (Table 4): BAX, CCNG1, CDKN1A, DDB2, EGFL7, FDXR, GDF15, LTBR, MDM2, TNFRSF10B (Additional file 4, Table S3). Most of these co-citations pertain to well-known pathways of TP53 regulation. For instance, MDM2 is a ubiquitin ligase and transcriptional target of TP53, CDKN1A (TP21) is a cyclin dependent kinase inhibitor and transcriptional target of TP53, BAX is a BCL2-associated protein involved in apoptosis. Fourth, the passenger genes are enriched with GO categories of caspace activation (hyper-geometric p-value $\leq$ 0.001 ), cyclin-dependent kinase activity ( $\mathrm{p}$-value $\leq$ $0.0025)$, cell cycle arrest ( $\mathrm{p}$-value $\leq 0.0069)$, and pathways of TP53 signaling (p-values $\leq 1.55 \times 10^{-5}, \leq 4.14 \times$ $10^{-4}$ ), and $\mathrm{G} 2 / \mathrm{M}$ checkpoint (p-value $\leq 0.0012$ ) (Table 5). In contrast, positive associations with TP53 mutations are not enriched with known TP53 targets or promoters containing TP53-binding motifs. Negative associations with TP53 mutations imply positive regulation of TP53 since most TP53 mutations are loss-offunction mutations.

161 mRNA expressions are negatively associated with DNA methylations of five gene clusters (Table 1). They include the association modules of PAX8 (61 passenger genes), BCR (58 passenger genes), HOXC13 (19 passenger genes), CCND1 and PPARG (13 passenger genes), and COL5A1 (10 passenger genes). The passenger genes of PAX8 and HOXC13 modules are enriched with the binding motifs of their drivers (PAX8: 34 of 61 genes, hyper-geometric p-value $\leq$ 0.0014, HOXC13: 12 of 19 genes, hyper-geometric $\mathrm{p}$-value $\leq 0.016$ ). Furthermore, a passenger gene in the CCND1/PPARG module, KLF4, is regulated by both CCND1 and PPARG from multiple previous studies (Additional file 4, Table S3).

241 mRNA expressions are negatively associated with two clusters of microRNA expressions (Table 1). Association module 81 possesses mir-92, mir-96, mir-106a, mir-20b, mir-17, mir-19b, mir-32, mir-135, mir-25, mir106b, mir-93, mir-106, mir-18, and mir-20 as drivers and 121 passenger genes. Association module 82 contains mir-24, mir-99b, mir-27b, mir-21, mir-125a, mir23a, mir-23b, and mir-27a as drivers and 120 passenger genes. The passenger genes in both modules are enriched with the predicted targets of their driver microRNAs (Table 3, association module 81: mir-96, pvalue $\leq 0.015, \operatorname{mir}-106 \mathrm{a}, \mathrm{p}$-value $\leq 0.046, \operatorname{mir}-17, \mathrm{p}$ - value $\leq 0.038$, mir-93, p-value $\leq 0.032$; association module 82: mir-21, p-value $\leq 0.001$ ). Therefore, some passenger mRNA expressions in these modules are probably regulated by their driver microRNAs.

\section{Gene clusters with coherent expression profiles are likely to} be regulated by transcription factors

A unique characteristic of NCI-60 mRNA expression data is the presence of a few large clusters with highly coherent expression profiles (Additional file 1, Figures S1, S2, S3, S4, S5, S6, S7, S8, S9, S10, S11, S12, S13). Two of the largest clusters are not associated with any observed molecular aberrations. We identified the transcription factors that were associated with the mRNA expressions in these clusters as candidates for intermediate regulators. These transcription factors may regulate the coherent expressions of downstream genes, yet the drivers that control the regulator expressions are unknown. Two association modules with intermediate regulators are reported in Table 1. Module 83 contains SMAD3, FOXD1, PLAU, BDNF, FOSL2, FOSL1 and RBMS1 as regulators and 338 passenger genes. Module 84 contains ERG, ELK4, NFATC1, RFX3, POU5F1 and ZNF350 as regulators and 338 passenger genes. The expression profiles of the two modules are shown in Figure 5.

Associations of regulators and passengers in these modules are supported by rich prior studies. Promoters of module 83 passengers are highly enriched with SMAD3-binding motifs (Table 3, 271 of 338 genes, hyper-geometric $\mathrm{p}$-value $\left.\leq 1.058 \times 10^{-7}\right)$. SMAD3 encodes a transcriptional modulator activated by transforming growth factor- $\beta$ (TGF $\beta$ ). Indeed, many passenger genes are members or downstream targets of the TGF $\beta$ signaling pathway according to previous studies, such as TGFBR2, ADAM12, CAV1, CCNB1, and EGFR (Table 6 and Additional file 4, Table S3). In addition, many passenger genes are co-cited with other candidate regulators (PLAU, BDNF, FOSL1) in previous publications. For instance, associations of BDNF and passenger genes such as ADAM17, AXL, and BCAT1 in neuron cells are previously reported (Additional file 4, Table S3).

Promoters of module 84 passengers are enriched with the binding motifs of NFATC1 (265 of 388 genes, hyper-geometric p-value $\leq 3.15 \times 10^{-5}$ ), POU5F (18 of 338 genes, hyper-geometric p-value $\leq 0.0027)$, ERG (67 of 338 genes, hyper-geometric p-value $\leq 0.0093)$, RFX3 (75 of 338 genes, hyper-geometric p-value $\leq 0.0481$ ), and ELK4 (76 of 338 genes, hyper-geometric p-value $\leq$ 0.0518). Many passenger genes are co-cited with some of these regulators in previous studies (Table 4).

Most association modules exhibit tissue-specific expressions NCI-60 cell lines constitute 9 distinct tissue origins. The patterns of molecular aberrations and gene expressions 


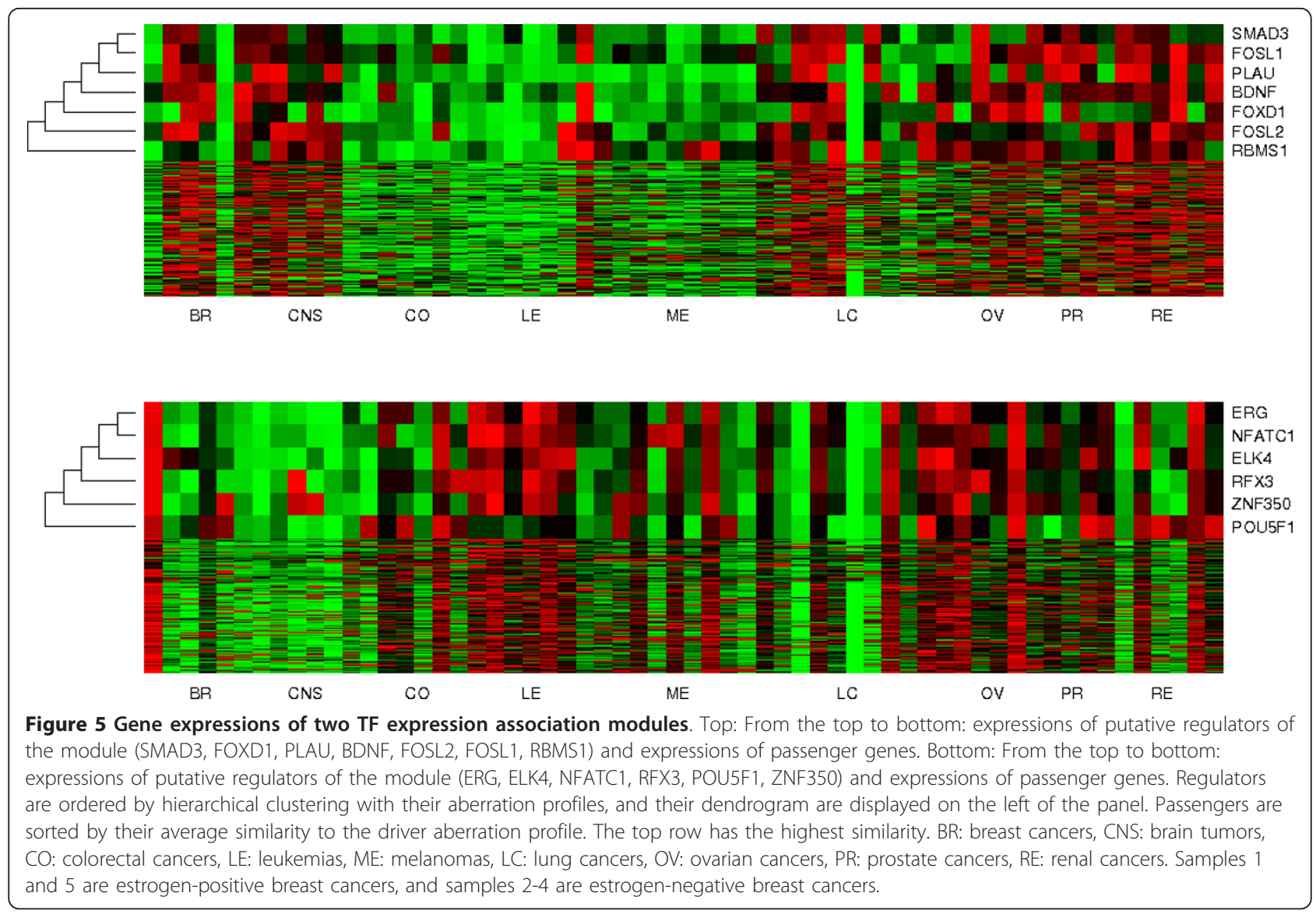

are likely to vary between tissue types. The inferred modules capture strong associations between drivers and passengers across all samples. Strong associations, however, can be manifested by either tissue-specific or tissue-independent patterns. These two types of patterns are illustrated in Figure 3. The driver of module 44 (segment $18 \mathrm{CNV}$ ) is amplified in melanoma cell lines alone, and its passenger expressions are up-regulated in melanoma cell lines. In contrast, the driver of module 66 (TP53 sequence) has prevalent mutations among all tissue types, and its passenger expression patterns are independent of tissue types. To check whether the effects of dysregulation are universal or tissue-specific, we extracted the tissue-specific patterns of drivers and passengers in each module (see descriptions in Materials and Methods and Additional file 1, Text S1) and visualized them in Figure 6. We observed the following intriguing results. First, about half of the cis-acting modules (internal segment CNVs as drivers) are coherently up or down regulated in at least one tissue type. In contrast, the majority of the trans-acting modules (all other types of drivers) have the same property. Second, despite many association modules possess tissue-specific patterns, none of them are coherently up or down regulated among all tissue types. In other words, a module has incoherent driver aberrations and passenger expressions in at least one tissue type. Third, the tissuespecific patterns are dominated by four tissue types: melanoma, leukemia, colon and central nervous system. For instance, 8 and 4 of 41 trans-acting modules contain up and down regulated passengers in leukemia respectively. 8 and 9 of 41 trans-acting modules contain up and down regulated passengers in melanoma respectively. 5 trans-acting modules contain differentially expressed passengers in renal cancers. In contrast, very few modules possess tissue-specific patterns on lung, ovarian or prostate cancers. Fourth, no module is coherently up or down regulated in breast cancer cell lines. Yet several of them possess coherent expressions in estrogen (ER)-positive or ER-negative samples. For instance, module 83 (Figure 5) passengers are up-regulated in ER-negative samples and down-regulated in ERpositive samples. Fifth, tissue-independent patterns belong to transacting modules with external segment CNVs or mutations as drivers. Five of 14 modules associated with mutations are tissue-independent: module 65 (CDKN2A), module 68 (TP53), module 70 (KRAS), module 74 (PIK3CA) and module 75 (PTEN). The 
Table 6 Summary information of association modules for microRNA expressions

\begin{tabular}{|c|c|c|c|c|c|}
\hline index & driver type & drivers & reg & sign & $\mathbf{N}$ \\
\hline 1 & inter CNV & seg 69 & SPOP,NME2,POLG2 & - & 38 \\
\hline 2 & inter CNV & $\operatorname{seg} 55$ & NR2C1,NFYB & - & 29 \\
\hline 3 & inter CNV & $\operatorname{seg} 51$ & RICS & + & 23 \\
\hline 4 & inter CNV & $\operatorname{seg} 18$ & $\mathrm{ZIC1} 1 \mathrm{MITF}$ & - & 19 \\
\hline 5 & inter CNV & $\operatorname{seg} 15$ & DNMT1,MBD3 & + & 15 \\
\hline 6 & inter CNV & seg 30 & SRF & + & 13 \\
\hline 7 & inter CNV & $\operatorname{seg} 66$ & MAP2K4 & - & 13 \\
\hline 8 & inter CNV & $\operatorname{seg} 68$ & BRCA1 & - & 12 \\
\hline 9 & inter CNV & $\operatorname{seg} 13$ & NFE2L2 & - & 10 \\
\hline 10 & mutation & TP53 & NA & + & 17 \\
\hline 11 & mutation & KRAS & NA & - & 15 \\
\hline 12 & mutation & APC & NA & + & 10 \\
\hline 13 & mutation & STK11 & NA & - & 10 \\
\hline 14 & methylation & $\mathrm{BCR}$ & NA & - & 12 \\
\hline 15 & $\mathrm{TF}$ & TF group & $1 \mathrm{NA}$ & + & 28 \\
\hline 16 & $\mathrm{TF}$ & MBD1 & NA & + & 19 \\
\hline 17 & $\mathrm{TF}$ & TFE3 & NA & + & 18 \\
\hline 18 & $\mathrm{TF}$ & BR2F1 & NA & + & 17 \\
\hline 19 & $\mathrm{TF}$ & GRLF1 & NA & + & 15 \\
\hline 20 & $\mathrm{TF}$ & HIF1A & NA & + & 14 \\
\hline 21 & $\mathrm{TF}$ & E4F1 & NA & + & 12 \\
\hline 22 & $\mathrm{TF}$ & TFDP1 & NA & + & 12 \\
\hline 23 & $\mathrm{TF}$ & FOXM1 & NA & + & 11 \\
\hline 24 & $\mathrm{TF}$ & ATF2 & NA & + & 10 \\
\hline 25 & intra CNV & chr14p & NA & + & 14 \\
\hline
\end{tabular}

Annotations follow Table 1. mutational states of these genes on the samples are not aligned with their tissue types. In contrast, modules associated with mutations of APC and BRAF possess tissue-specific patterns, as APC and BRAF mutations occur primarily on colon cancer and melanoma samples respectively. Similarly, the majority of microRNA association modules possess tissue-specific patterns, and these patterns are dominated by up/down regulations in leukemias, central nervous systems and colons. Different from mRNA modules, only two microRNA modules possess melanoma-specific patterns. The tissue-specific patterns of microRNA modules are displayed in Additional file 1, Figure S17.

\section{MicroRNA expressions are driven by distinct aberration mechanisms from mRNA expressions}

195 of 303 microRNA expressions are associated with observed molecular aberrations. We grouped these associations into 25 modules and summarized their information in Table 6. Intriguingly, the drivers of the topranking microRNA modules are marginally overlapped with those of the top-ranking mRNA modules, suggesting that microRNA and mRNA expressions are driven by distinct molecular aberrations. The complete list of association modules are reported in Additional file 3, Table S2, and driver aberrations and passenger microRNA expressions are visualized in Additional file 1, Figures S14, S15, S16.

Table 2 shows the FDRs for each type of associations and all association together for microRNA modules. The

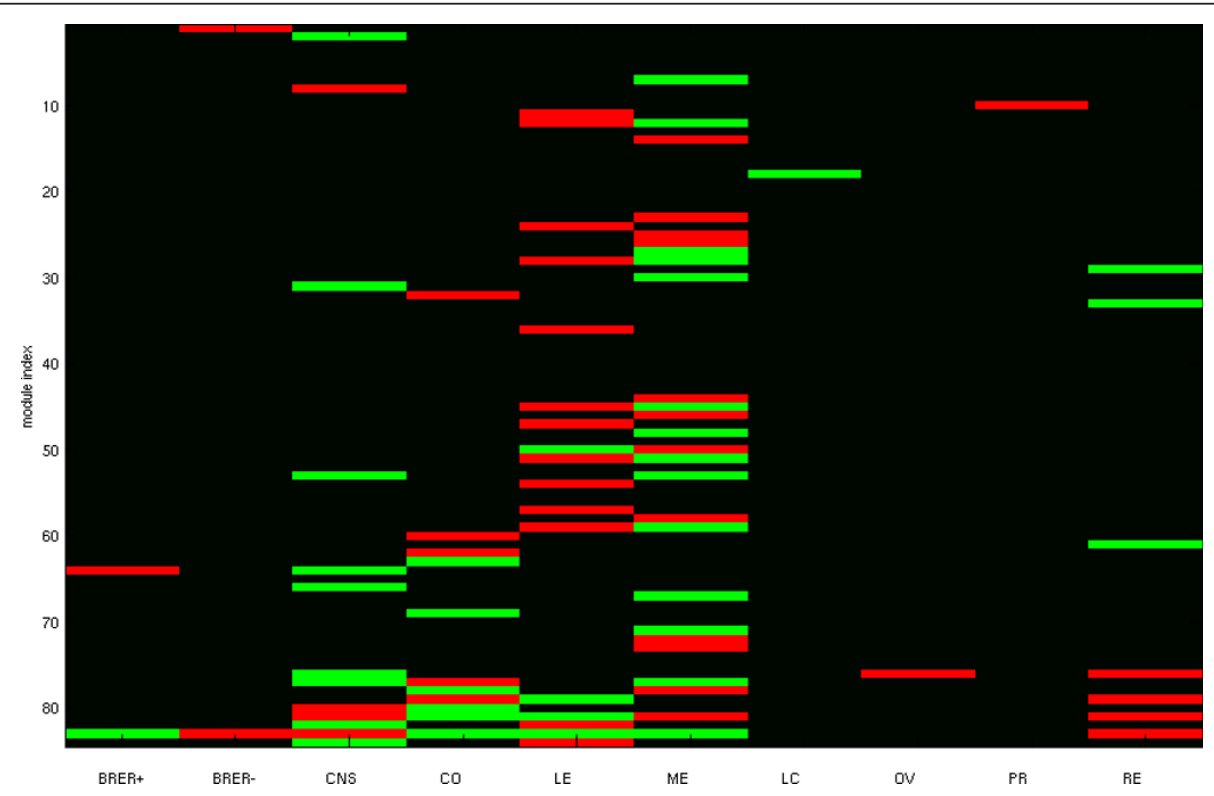

Figure 6 Tissue-specific patterns of mRNA association modules. Each row represents the tissue-specific pattern of a module. Red: coherent up-regulation in a tissue type. Green: coherent down-regulation in a tissue type. Black: incoherent expressions in a tissue type. Cis-acting modules range from 1 to 43. Trans-acting modules range from 44 to 84. BRER+: ER-positive breast cancers, BRER-: ER-negative breast cancers, CNS: brain tumors, CO: colorectal cancers, LE: leukemias, ME: melanomas, LC: lung cancers, OV: ovarian cancers, PR: prostate cancers, RE: renal cancers. 
microRNA FDRs exhibit same trend as the mRNA FDRs. Associations with segment CNVs yield the lowest rates, followed by associations with mutations and transcription factor expressions. Associations with DNA methylation yield the worst performance. Associations with microRNA expressions and local segment CNVs were not considered. The overall FDRs calculated by the two methods are 0.292 and 0.394 respectively.

Information about regulators and functions of microRNAs is much more scattered than those of proteincoding genes. We incurred a batch search on PubMed to identify co-citations of drivers/regulators and passenger microRNAs in these modules. Overall, co-citations of drivers/regulators and passengers were found in 9 associations modules. Table 4 and Additional file 4, Table S3 report the co-citation outcomes for microRNA modules.

\section{Cis-acting effects with segment CNVs are absent on microRNAs}

A remarkable distinction between mi-croRNA and mRNA associations is the lack of associations with local segment CNVs in microRNA modules. Mi-croRNA expressions are poorly correlated with their local segment CNVs compared to mRNA expressions (Additional file 1, Figure S18). The only possible exception is a module of microRNAs located on chromosome 14p (Additional file 3, Table S2). These microRNAs exhibit a coherent expression pattern (Additional file 1, Figure S16) and are localized on chromosome 14p. Thus they are likely to be modulated by local segment CNVs. Yet we cannot verify these associations since chromosome $14 \mathrm{p}$ is not covered by the CNV data.

\section{Associations suggest the existence of feedback loops} between microRNAs and protein-coding genes

Co-citation search outcomes demonstrate bidirectional regulatory links between the driver/regulator genes and passenger mircroRNAs. Table 7 summarizes these regulatory links reported from prior studies. Most drivers/ regulators of these microRNA modules are known or predicted targets of the passenger microRNAs. Hence there are possible bidirectional links connecting transcription factors and microRNAs. In some cases, bidirectional links are reported. For instance, MAP2K4 in module 7 is both a known regulator and target of two passenger microRNAs mir-25 and mir-92. Similarly, TP53 in module 10 is both a known regulator and target of two passenger microRNAs mir-15a and mir-16.

\section{Discussion}

Uncovering causal relations of genes from static, observational data alone is challenging due to the ambiguities of transferring associations into causal information. We address this problem by exploiting two widely accepted hypotheses in cancer genomics: (1)molecular aberrations on DNAs (mutations, copy number variations, DNA methylations) affect the levels of RNA transcripts but not the other way around, (2)if both cis-acting and trans-acting effects of segment CNVs are detected, then cis-acting effects are more likely to be the direct causes for expression changes. Based on these hypotheses we built association modules between putative driver molecular aberrations and passenger mRNA or microRNA gene expressions. Our predictions are supported by various validation results. First, the downstream targets of several well-known cancer-related genes such as MITF, TP53 and APC are retrieved from our association modules. Second, the passenger genes of large modules are enriched with the putative targets of their regulators or drivers. Third, putative targets of MYB are down-regulated under the MYB siRNA treatment. Fourth, associations between molecular aberrations and microRNA expressions reveal the bidirectional regulatory links between transcription factors and microRNAs.

One intriguing observation is that each type of molecular aberrations participate in at least one association module. Therefore, to track the causes of expression variations it is necessary to interrogate multiple types of molecular aberrations concurrently. NCI-60 datasets are sparse in mutations (24 valid genes), copy number variations (219 valid genes) and DNA methylations (320 valid

Table 7 Regulatory links between drivers/regulators and microRNAs

\begin{tabular}{llllll}
\hline index & link & PMID & index & link & PMID \\
\hline 4 & mir-182, mir-183 $\rightarrow$ MITF & 17597072 & 15 & mir-21 $\rightarrow$ SMAD3 & 19816956 \\
4 & mir-130b $\rightarrow$ ZIC1 & 20676061 & 15 & mir-23a $\rightarrow$ SMAD3 & 18508316 \\
5 & DNMT1 $\rightarrow$ let-7a & 17308078 & 15 & mir-24 $\rightarrow$ SMAD3 & 18353861 \\
6 & SRF $\rightarrow$ mir-143 & 21069820 & 15 & mir-10a $\rightarrow$ BDNF & 20309390 \\
7 & mir-25 $\leftrightarrow$ MAP2K4 & 19861690,15652477 & 15 & mir-125b $\rightarrow$ BDNF & 19635812 \\
7 & mir-92 $\leftrightarrow$ MAP2K4 & 17683260,15652477 & 15 & mir-134 $\rightarrow$ BDNF & 20622856,16421561 \\
10 & mir-15a, mir-16 $\leftrightarrow$ TP53 & 19347736,15652477 & 15 & mir-210 $\rightarrow$ BDNF & 19826008 \\
14 & mir-203 $\rightarrow$ BCR & 18538733 & 20 & mir-34c $\rightarrow$ HIF1A & 20861672 \\
\hline
\end{tabular}

Index: microRNA association module index. Link: regulatory links between regulators and microRNAs. Arrows indicate the directions of regulation. PMID: the PubMed ID of the reported link. 
genes). Consequently, about half of the mRNA expression data (3571 genes) are not significantly associated with observed aberrations. This drawback will become less prominent in new generations of comprehensive cancer genomics projects (e.g., TCGA [7] and ICGC [8]), as they employ high density microarrays and nextgeneration sequencing to cover the entire genomes.

Associations with driver molecular aberrations can be extended to clinically relevant phenotypes such as drug resistance [51]. Unlike mRNA or microRNA expressions, these phenotypes are not the direct products of molecular aberrations. Thus associations are likely to be mediated by the genes functionally related to the phenotypes. For instance, from the drug response data on NCI-60 cell lines [19] we found leukemia cell lines were sensitive to the majority of compounds tested (data not shown). This observation coincides with up-regulation of genes involved in transcriptional and translational processes in leukemia (Additional file 5, Table S4). Both drug responses and gene expressions reflect the high growth and division rates of leukemia cell lines relative to other samples.

Two of the largest association modules (modules 83 and 84) lack driver aberrations but consist of intermediate regulators. In particular, multiple lines of evidence indicate the expressions of module 83 are controlled by TGF $\beta$ pathway activities. Yet it is unclear which molecular aberrations modulate the expressions of the intermediate regulators. Examination of the mutations and DNA methylations of members on the TGF $\beta$ pathway may reveal their driver aberrations.

Cis-acting and trans-acting effects with segment CNVs dominate the association modules of mRNA expressions (61 of 84 association modules). Amplifications and deletions of chromosomal segments are powerful driver aberrations, as they can possibly affect many genes on the segments and downstream targets on other loci. Determination of cis-acting and trans-acting effects with CNVs remains an open problem. We observed a tendency of cis-acting genes to cluster on certain hotregions of chromosomes. Yet many cis-acting genes are still scattered around the entire chromosomes. The distributions of cis and trans-acting genes will become more clear from the data of high density CGH arrays.

Associations with mutations of several cancer-related genes - TP53, CDKN2A, APC - are largely consistent with their known targets. However, the passenger sets of positive and negative associations exhibit differential levels of enrichment. Passenger genes negatively associated with TP53 mutations (module 66) are enriched with TP53-binding motifs (Table 3 ) and known targets of TP53 (Table 5 and [50]). In contrast, passenger genes positively associated with TP53 mutations (module 68) do not possess the enrichment. TP53 undergoes loss-of- function mutations, thus negative associations imply positive regulation between TP53 and its targets. Although negative regulation of TP53 has been previously reported [52], on NCI-60 TP53 seems to be an activator of its prominent direct targets. The enriched functional information is reversed in APC and CDKN2A mutations. 15 of the passenger genes positively associated with APC mutations (module 62) are involved in the Wnt pathway or associated with APC. In contrast, only 3 of the passenger genes negative associated with APC mutations (module 63) are related to APC. Similarly, 5 of the passenger genes positively associated with CDKN2A mutations (module 69) are related to CDKN2A, whereas only 2 of the passenger genes negatively associated with CDKN2A mutations (module 65) are related to CDKN2A. Unlike TP53, APC and CDKN2A are inhibitors of signaling pathways (Wnt and cell cycle control) and possess no direct targets. Hence their loss-of-function mutations would activate the downstream targets of the pathways. In contrast, genes down-regulated in APC or CDKN2A mutants are likely to arise from secondary effects instead of direct consequences of APC or CDKN2A mutations.

Two large clusters of mRNA expressions are negatively associated with groups of microRNA expressions (modules 81 and 82 ). The causality between microRNAs and mRNAs are ambiguous since regulation can take place in both directions. The passenger genes are enriched with the predicted targets of driver microRNAs. Additional evidence is required to determine their causal directions.

In NCI-60 cell lines, microRNA and mRNA expressions seem to be driven by distinct molecular aberrations. Unlike mRNA expression modules, none of the microRNA expression modules are associated with local segment CNVs. This is curious since many microRNAs in this study are located on the partitioned segments and between the protein-coding genes possessing the cis-acting effects with segment CNVs. Poor correlations between microRNA expressions and their local segment CNVs suggest either the low resolution of the CGH data or the trans-acting nature of microRNA regulation.

It is more difficult to validate the association modules of microRNA expressions due to the paucity of regulatory and functional information. Intriguingly, we found that many drivers and regulators were known or predicted targets of their passenger microRNAs (Table 7). In some cases, regulation along both directions between drivers and passengers is reported (for instance, MAP2K4 v.s. mir-25 and mir-92). The association outcomes and prior studies suggest feedback regulation between driver transcription factors and passenger microRNAs. 
It is worth noting that pairwise associations inferred from the data are projections of multi-variate models from drivers to the target passenger. A driver links to a passenger if their association cannot be explained by other driver variables. Therefore, "pairwise associations" in this study carry a very different meaning from purely pairwise scores such as correlation coefficients. A more adequate analogy is conditional independence in probabilistic graphical models. A driver A links to a passenger $\mathrm{X}$ if $\mathrm{A}$ is not conditionally independent of $\mathrm{X}$ given any other driver variable. Such direct and indirect relations can be captured by a variety of prior approaches such as Bayesian networks, mutual information and regression. Our contribution is not to invent/introduce a new mathematical tool to tackle the same problem, but to develop a data integration framework to exploit the statistical and mechanistic properties of the data. More specifically, the novelty of this work includes (1)Prioritize the order of adding association links according to mechanistic information. Cis-acting effects are first considered as they provide direct explanation for passenger expression fluctuations. Trans-acting effects with aberrations on DNA as drivers (CNV, mutations, DNA methylations) are taken into account only if they provide additional explanatory power relative to cis-acting effects. Finally, transacting effects without DNA aberrations (microRNA or transcription factor expressions) are introduced on top of the lower layered models. (2)Project the complex network of conditional independence relations onto pairwise links and group these links as driver-centric association modules. This simplification is useful and practical as it is much easier to examine and validate a coherent module than a complex network.

Projecting a complex regulatory network onto wellstructured modules certainly yields information loss. Genes are often regulated by combinatorial interactions of multiple transcription factors, chromatin modifiers and signaling proteins. Moreover, the regulatory programs may vary with tissue types. In this work, we chose to ignore combinatorial interactions and tissuespecific regulatory programs and focused on strong marginal effects manifested on the variations across all 60 cell lines. This simplification seems necessary given the limited size of NCI-60 data. A new generation of cancer genomic data - such as TCGA and ICGC - often cover much more patients with a specific tumor type. Therefore, it is possible to include the combinatorial interactions and tissue-specific regulations in an extension of the current models.

The FDR values clearly depend on the constraints of associations. Internal segment $\mathrm{CNVs}$ require the physical proximity of drivers and passengers, and external segment CNVs require the existence of intermediate regulators linking segment $\mathrm{CNVs}$ and passenger expressions. These additional constraints push the FDRs below significant levels. In contrast, modules of mutations, DNA methylations, microRNA and TF expressions as drivers rely only on associations between drivers and passengers. Thus their FDRs are substantially higher than standard significant values. To lower the FDRs on those modules, additional constraints such as evidence of physical interactions between drivers and passengers are probably needed. However, since the FDRs reported in Table 2 are the aggregate results over all modules of the same type, the high values do not deteriorate the relevance of individual modules. For instance, multiple lines of evidence support the modules associated with TP53 mutations, CDKN2A mutations, PAX8 methylations, as well as TF groups 1 and 2 expressions. It is difficult to discriminate these biologically relevant modules from potentially spurious modules from the NCI-60 data alone. External information or experimental validation has to be incorporated. In this work, we use external information and experimental validation to justify the models inferred from data alone. A refined version of the model construction algorithm should include these additional information in the loop.

Only 5 transcription factor binding motifs are significantly enriched on module passengers after Bonferroni correction (TBP, PAX8, SMAD3, NFATC1 and POU5F). These unsatisfactory results can be attributed to both the conservative nature of Bonferroni correction and the accuracy of motif presence as a proxy for regulation. We sought the presence of motifs within $5 \mathrm{~kb}$ upstreams of the transcription start sites. This simple scheme may create many false positives and false negatives. For instance, only 27 of the 113 experimentally validated MITF targets [45] contain the MITF-binding motif within $5 \mathrm{~kb}$ promoters, and only 13 of the 67 experimentally validated TP53 targets [50] contain the TP53-binding motif within $5 \mathrm{~kb}$ promoters. The discrepancy between motif presence and experimentally validated targets may partially explain the relatively high motif enrichment p-values of modules 44 (MITF) and 66 (TP53). Restrictions to promoters with multiple motif occurrences reduce false positives but increase false negatives. The enrichment results are worse than Table 3 (Additional file 9, Table S8). Consequently, we should treat motif enrichment analysis as one error-prone validation and use it together with other validations (cocitations, functional category or pathway enrichments). Other types of validations are also subjected to error. It remains an open problem to systematically validate the large-scale models inferred from high-throughput data.

In this work, we built association modules from NCI60 data alone and attempted to validate the inferred modules with external information. An alternative and common approach is to start with prior models using 
external information (e.g., pathways, GO categories, protein-DNA and protein-protein interactions) and learn the models explained by the data. As a simple comparison, we treated each pathway and GO category as the passenger gene set of a module and identified the drivers that significantly explained their expressions. The results are reported in Additional file 10, Table S9. Overall, only a small number of GO categories and pathways are significantly explained by driver aberrations in NCI-60 data. Prominent ones include genes involved in melanosome and melanin biosynthesis and explained by MITF transcription factor expressions, ribosome genes explained by a cluster of 9 microRNAs, and members of proteasome genes explained by internal segment CNVs on chromosome 14q. Notice that genes involved in melanosome and melanin biosynthesis substantially overlap with module 44 .

\section{Conclusions}

We proposed a computational method to build associations between molecular aberrations (drivers) and mRNA/microRNA expressions (passengers) and grouped the genes/microRNAs into modules according to their associated molecular aberrations. Intriguingly, a considerable portion of these associations implied causal relations according to in-silico and experimental validations. We found passengers in these association modules were enriched with the putative targets of the drivers, functional categories and pathways in which the drivers were involved, and regulatory/association links confirmed by prior publications. Moreover, we demonstrated that the predicted MYB targets were down-regulated in a MYBsiRNA treated leukemia cell line. The reported association modules indicated that gene/microRNA expressions in cancer were driven by diverse aberration mechanisms including copy number variations, mutations, DNA methylations, microRNA and transcription factor expressions. In addition, the results suggested distinct driver mechanisms between mRNA and microRNA expressions and the existence of bidirectional regulatory links between microRNAs and transcription factors.

\section{Methods}

\section{Data sources and processing}

NCI-60 constitutes a panel of 60 cell lines derived from 9 tissue types: breast ( 5 cell lines), central nervous system ( 6 cell lines), colon ( 7 cell lines), leukemia ( 6 cell lines), melanoma (10 cell lines), lung (9 cell lines), ovary (7 cell lines), prostate ( 2 cell lines) and kidney (8 cell lines). Melanoma cell line MDA-N is derived from MDA-MB435, thus the two cell lines are highly similar. Among the 5 breast cancer cell lines 2 are estrogenpositive (MCF7 and T47D) and the remaining 3 are estrogen-negative (MDA-MB-231, HS578T. BT-549).
Seven datasets of NCI-60 cell lines were downloaded from the website of the Genomics and Bioinformatics Group at NCI (GBC): mutation analysis of 24 cancer genes [10], Comparative Genomic Hybridization (CGH) array data of DNA copy number variations [11], cytosine methylation profiling on promoters [12], cDNA microarray data [15], Affymetrix transcript profile data [16], and Agilent transcript profile data [17] of mRNA expressions, and microRNA expression data [13]. The union of these datasets covered 14856 genes and 303 microRNAs.

Continuous data (CNVs, mRNA expressions, microRNA expressions, DNA methylations) were first ranktransformed into cumulative distribution function (CDF) values and then converted into probability vectors of trinary states using probabilistic quantization [33]. This transformation normalizes the data in the same scale and preserves the information from continuous data. Discrete data (mutations) was directly fed into the model without processing. Detailed procedures of data normalization and combination are reported in Additional file 1, Text S1.

Spatial dependency of CNV data was manifested from the measurements of 219 genes in a CGH array [33]. Adjacent probes on the same chromosome tend to have higher correlations than randomly selected probes. To exploit the spatial dependency of CGH probes, we devised a recursive algorithm to partition each chromosome into correlated segments using the $\mathrm{CNV}$ data. The algorithm is described in Additional file 1, Text S1. 86 segments were obtained from the $\mathrm{CNV}$ data and reported in Additional file 6, Table S5. The CNV data of a segment is the mean over the CNV data of all its constituent probes.

\section{Logistic regression models}

We used logistic regressions to model the effects of molecular aberrations on gene or microRNA expressions. Denote $y$ the expression of a gene or microRNA and $x$ the driver aberrations that explain $y$. The conditional probability is

$$
P(y \mid x)=\frac{1}{Z(x)} e^{\sum_{i} \lambda_{i} f_{i}(x) y}, \lambda_{i} \geq 0, \forall i .
$$

$f_{i}(x)$ 's are scalar feature functions specifying the relations of $x$ and $y . \lambda_{i}$ 's are nonnegative parameters, and $Z$ $(x)$ is the partition function that normalizes the conditional probabilities. In this work $f_{i}(x)$ 's are linear functions of feature values. $f_{i}\left(x_{i}\right)=x_{i}$ if aberration $x_{i}$ activates expression $y, f i\left(x_{i}\right)=-x_{i}$ if aberration $x$ represses expression $y$.

Given observed data $D$ and two nested models $M_{0}, M_{1}$ $\supseteq M_{0}$, we incurred a standard hypothesis testing procedure to calculate the log-likelihood ratio and p-value of 
$D$. The pairwise scores measured the goodness of fit of $M_{1}$ against the null model $M_{0}$. Furthermore, given the models $M_{1}$ and $M_{2}$ of two candidate driver aberrations we tested the joint model $M_{12}$ against $M_{1}$ and $M_{2}$ respectively. The testing results provide information whether the explanatory power of one model can be covered by another. Detailed procedures of parameter estimation, evaluation of log likelihood ratios and pvalues as well as model selection are described in Additional file 1, Text S1.

\section{Building association modules}

An association module consists of a small number of bona fide driver molecular aberrations and a subset of passenger genes or microRNAs. The expression profiles of passengers are explained by fluctuations of drivers. For trans-acting effects with segment CNVs, the associations between drivers and passengers are mediated by regulators on the segments. We require that a valid association module should satisfy the following conditions. First, a driver explains each passenger expression with a significant pairwise score. Second, a driver provides an explanatory power that cannot be replaced by any other drivers. Third, cisacting effects have a higher priority than associations with non-local aberrations. Fourth, a module has at least 10 passengers. Toward this goal the association modules were constructed by the following procedures.

1. For each passenger mRNA or microRNA expression, evaluate the pairwise association scores with all candidate driver aberrations. Keep the associations that pass the threshold values of these scores.

2 . If a passenger gene or microRNA is associated with an external segment $\mathrm{CNV}$, then find the transcription factor mediating the associations between external segment CNVs and passenger expressions.

3. Rule out a transcription factor as a candidate driver for the modules of regulatory effects if the transcription factor is associated with any observed molecular aberration.

4. For each passenger mRNA or microRNA expression, incur model selection to rule out the driver aberrations that can be replaced by other drivers. Briefly, for the models $M_{1}$ and $M_{2}$ of two candidate driver aberrations, test the joint model $M_{12}$ against $M_{1}$ and $M_{2}$ respectively. If $M_{12}$ is significantly better than $M_{1}$ but does not outperform $M_{2}$, then remove $M_{1}$. Apply this filtering procedure for all pairs of driver aberrations.

5. Group passenger genes or microRNAs into modules by their drivers. Report the modules with $\geq 10$ passenger members. Passengers of distinct association modules may overlap.

Detailed procedures for building association modules are described in Additional file 1, Text S1.

\section{Experimental validation \\ Selection of putative targets and control genes of MYB}

To validate the causal implications of association outcomes we selected several putative targets of MYB and measured their expression responses with and without the treatment with an MYB siRNA. Putative MYB targets satisfy the following criteria: (1)their mRNA expressions were positively associated with segment $31 \mathrm{CNV}$, (2)their mRNA expressions were positively associated with MYB expression in NCI-60 data under normal conditions, (3)their mRNA expressions were positively associated with MYB expression in another expression dataset of NCI-60 cell lines exposed under radiation ([53], Additional file 1, Figure S19), (4)their mRNA expressions were positively associated with MYB in a dataset containing 73 normal tissues [54]. 31 genes passed these filtering criteria. Among them we then selected 11 genes based on the constraints of primer design: CPSF6, CTCF, HCLS1, KHDRBS1, NFATC3, ORC1L, PAICS, POLD3, RBMX, SMC1A, and ZNF131.

In addition to predicted MYB targets we also selected several control genes which were constituently expressed across the NCI-60 cell lines, regardless of the MYB expression levels. From the top 10 candidates we selected 6 based on the constraints of primer design: SSR2, GNB2, HSPC152, EIF4A1, RPS15, HINT1. Detailed procedures of selecting putative MYB targets and control genes are described in Additional file 1, Text S1.

\section{siRNA treatments and RNA measurements}

Human erythroleukemia cells (K562) were purchased from NCI (Frederick, MD) and maintained in RPMI 1640 medium (Invitrogen, CA). The sequences of siRNAs were adopted from previous reports: siRNA against c-myb (c-myb siRNA); sense: 5-UGUUAUUGCCA AGCACUUAAA -3; anti-sense: 5-UAAGUGCUUGGCAAUAACAGAA -3; siRNA against GFP (control siRNA): sense sequence: 5-UGCGCUCCUGGACGUAGCCTT-3; antisense: 5-GGCUACGUCCAGGAGCGCATT-3.

siRNA transfection was performed as described previously with minor modifications. Briefly, cells were seeded at a density of $2 \times 10^{5}$ cells/well in a 6-well plate with $2 \mathrm{~mL}$ culture medium per well. Right after the seeding, the cells were transfected with c-myb siRNA or control siRNA using LipofectAMINE 2000 (Lf 2000, Invitrogen, CA). The siRNA/Lf2000 complex was then 
incubated with the cells ( $\mathrm{nM})$ for $48 \mathrm{~h}$ and the cells were harvested for further assays.

RNA isolation and cDNA synthesis were demonstrated according to the manufacturers protocols. Briefly, total RNA of the treated K562 cells was isolated and then converted to cDNA by incubating $1 \mu \mathrm{g}$ RNA with $2.5 \mu \mathrm{M}$ Oligo(dT)23, $500 \mu \mathrm{M}$ dNTP and $1 \mu \mathrm{l}$ of RNase Inhibitor (New England Biolabs, Beverly, MA), 5 $\mathrm{mM}$ DDT, $4 \mu \mathrm{l}$ of 5 First-Strand buffer and $1 \mu \mathrm{l}$ of SuperScript III Reverse transcriptase (Invitrogen) for $1 \mathrm{~h}$ at 50 degrees Celsius in a total volume of $20 \mu \mathrm{l}$ in a microtube.

Real-time PCR was performed in a Mastercycler epgradient-S thermocycler (Eppendorf, Hamburg, Germany) with FastStart TaqMan Probe Master (ROX) and Universal ProbeLibrary (Roche Diagnostics GmbH, Manheim, Germany) according to the manufacturers instructions. The primers and the probe used for detecting each gene are listed in Additional file 8, Table S7. The amplification conditions were $10 \mathrm{~min}$ at 95 degrees Celsius, followed by 40 cycles of 95 degrees Celsius for $15 \mathrm{~s}$ and 60 degrees Celsius for $1 \mathrm{~min}$. The quantity was determined from the experimental threshold cycle on a standard curve of the data from a series of serial dilution of the mixture of generated cDN A. The mRN A level of the gene of interest was normalized by that of ACTB ( $\beta$-actin) as an endogenous control. Detailed procedures of RNA quantification are described in Additional file 1, Text S1. The measured data of qRT-PCR are reported in Additional file 7, Table S6.

\section{In-silico validation \\ Evaluation of false discovery rates}

For each type of association modules, we randomly permuted aberration and expression data 1000 times and estimated the empirical distribution of the number of significant pairwise associations arising from permuted data. The expected number and the 99 percentile number of false positives were calculated from this empirical distribution. The number of positive calls from the data was the number of significant pairwise associations. Two types of false discovery rates were evaluated accordingly:

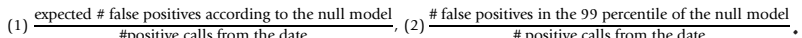

Enrichment analysis of putative targets on passenger genes 19 transcription factors appear in both drivers/regulators of the association modules and the TRANSFAC database [37]. We extracted their binding motifs from TRANSFAC and $5 \mathrm{~kb}$ promoter sequences of 27748 human genes from the UCSC Genome Browser [55]. For each transcription factor, the occurrences of its binding motif on all promoters and on the passenger promoters were counted. A standard Fisher's exact test was applied to evaluate the significance of motif enrichment on passenger genes. Motif search and Fisher's exact test were implemented by our own $\mathrm{C}$ programs.

Two association modules of mRNA expressions contain microRNA expressions as drivers. We extracted the putative targets of the driver microRNAs from the union of three databases: TargetScan [38], microRNA. org [39], and miRBase [40]. Enrichment analysis was carried out on the passenger genes of these modules.

\section{Co-citation analysis on PubMed database}

We incurred a batch search on the PubMed database to find all the pairs of drivers/regulators and passengers that were co-cited in the same publications. The spurious results from the automated search were removed by human inspection. Manual curation also identified the pairs conferring regulatory or association relations. To assess the confidence of co-citation outcomes, for each module we replaced passengers with random genes or microRNAs and counted co-cited pairs. The maximum numbers of co-cited pairs over 10 random trials are reported in Table 4.

\section{Enrichment analysis of functional categories and pathways}

We extracted 4822 functional categories from the Gene Ontology database [41] and 889 pathways from three pathway databases: Reactome [43], BioCarta [44], and the NCI Pathway Interaction Database [42]. For each association module, we applied standard a Fisher's exact test to identify enriched GO categories and pathways for the passenger genes.

\section{Extraction of tissue-specific patterns}

We extracted tissue-specific patterns of association modules with the following procedures. First, we obtained the tissue-specific pattern for each mRNA expression profile. An expression profile was written as a linear combination of "ideal" tissue-specific expression profiles, and the up/down regulation on a tissue type was determined by its mixture coefficient. An ideal tissue-specific expression profile has 1s on samples belonging to the target tissue and 0s on all other samples. Second, for each association module we checked whether its passengers were enriched with tissue-specific genes of each tissue type. Third, we employed gene set enrichment analysis [21] to find tissue-specific patterns of drivers. Finally we reported the intersection of tissuespecific patterns of drivers and passengers. Detailed procedures are elaborated in Additional file 1, Text S1.

\section{Additional material}

Additional file 1: Text S1 includes procedures of data processing, model selection, experimental and in-silico validations, heatmap visualizations of molecular aberrations and $\mathrm{mRNA} /$ microRNA 
expressions of association modules, and distributions of correlation coefficients between segment CNVs and their constituent genes and microRNAs.

Additional file 2: Table S1 reports the association modules of mRNA expressions

Additional file 3: Table S2 reports the association modules of microRNA expressions

Additional file 4: Table S3 reports the co-cited driver/regulatorpassenger pairs and the PubMed IDs of the citations in association modules.

Additional file 5: Table S4 reports the enriched GO categories and pathways for each association module.

Additional file 6: Table S5 reports the information of partitioned segments

Additional file 7: Table S6 reports the expression responses of putative targets and control genes in c-myb siRNA experiments

Additional file 8: Table S7 reports the primer sequences for RT-PCR

in c-myb siRNA experiments.

Additional file 9: Table S8 reports the enrichment of driver/ regulator binding motifs on passenger promoters with multiple motif occurrences.

Additional file 10: Table S9 reports the association outcomes between candidate drivers and GO categories/pathways.

\section{Acknowledgements}

We thank Ker-Chau Li, Kung-Hao Liang and Pei-Ing Hwang for the inputs and advice during the preparation of the manuscript. CHY is supported by the Institute of Statistical Science of Academia Sinica, the National Science Council grants of the Republic of China, Taiwan (grant numbers 98-2118-M001-025-MY2 and 100-2118-M-001-008-MY2).

\section{Author details}

'Institute of Statistical Science, Academia Sinica, Academia Road, Sec 2, Taipei, Taiwan. ${ }^{2}$ Ontario Institute for Cancer Research, 101 College Street, Toronto, Canada. ${ }^{3}$ Nagoya City University, Nagoya, Japan.

\section{Authors' contributions}

$\mathrm{CHY}$ conceived and encoded the association algorithms and performed computational analysis on NCl-60 data. SDL and TT performed the siRNA experiments on MYB targets. YFH performed co-citation analysis on inferred modules. All authors read and approved the final manuscript.

Received: 6 June 2011 Accepted: 4 November 2011

Published: 4 November 2011

\section{References}

1. Davies H, Hunter C, Smith R, Stephens P, Greenman C, Bignell G, Teague J, Butler A, Edkins S, Stevens C, Parker A, O'Meara S, Avis T, Barthorpe S, Brackenbury L, Buck G, Clements J, Cole J, Dicks E, Edwards K, Forbes S, Gorton M, Gray K, Halliday K, Harrison R, Hills K, Hinton J, Jones D, Kosmidou V, Laman R, Lugg R, Menzies A, Perry J, Petty R, Raine K, Shepherd R, Small A, Solomon H, Stephens Y, Tofts C, Varian J, Webb A, West S, Widaa S, Yates A, Brasseur F, Cooper CS, Flanagan AM, Green A, Knowles M, Leung SY, Looijenga LH, Malkowicz B, Pierotti MA, Teh BT, Yuen ST, Lakhani SR, Easton DF, Weber BL, Goldstraw P, Nicholson AG, Wooster R, Stratton MR, Futreal PA: Somatic mutations of the protein kinase gene family in human lung cancer. Cancer Research 2005 65(17):7591-7595.

2. Wood LD, Parsons DW, Jones S, Lin J, Sjoblom T, Leary RJ, Shen D, Boca SM, Barber T, Ptak J, Silliman N, Szabo S, Dezso Z, Ustyanksky V, Nikolskaya T, Nikolsky Y, Karchin R, Wilson PA, Kaminker JS, Zhang Z, Croshaw R, Willis J, Dawson D, Shipitsin M, Willson JK, Sukumar S, Polyak K, Park BH, Pethiyagoda CL, Pant PV, Ballinger DG, Sparks AB, Hartigan J, Smith DR, Suh E, Papadopoulos N, Buckhaults P, Markowitz SD, Parmigiani G, Kinzler KW, Velculescu VE, Vogelstein B: The genomic landscapes of human breast and colorectal cancers. Science 2007 318:1108-1113.

3. van't Veer $L$, Dai $H$, van de Vijver MJ, He YD, Hart AA, Mao M, Peterse HL, van der Kooy K, Marton MJ, Witteveen AT, Schreiber GJ, Kerkhoven RM, Roberts C, Linsley PS, Bernards R, Friend SH: Gene expression profiling predicts clinical outcome of breast cancer. Nature 2002, 415(6871):530-536.

4. Blenkiron C, Goldstein LD, Thorne NP, Spiteri I, Chin SF, Dunning MJ, Barbosa-Morais NL, Teschendorff AE, Green AR, Ellis IO, Tavar S, Caldas C, Miska EA: MicroRNA expression profiling of human breast cancer identifies new markers of tumor subtype. Genome Biology 2007, 8:R214.

5. Frohling S, Dohner H: Chromosomal Abnormalities in Cancer. The New England Journal of Medicine 2008, 359:722-734.

6. Jones PA, Baylin SB: The epigenomics of cancer. Cell 2007, 128:683-692.

7. The Cancer Genome Atlas Research Network: Comprehensive genomic characterization defines human glioblas-toma genes and core pathways. Nature 2008, 455(7216):1061-1068.

8. The International Cancer Genome Consortium: International network of cancer genome projects. Nature 2010, 464(7291):993-998.

9. Roschke AV, Tonon G, Gehlhaus KS, McType N, Bussey KJ, Lababidi S, Scudiero DA, Weinstein JN, Kirsch IR: Karyotypic complexity of the NCl-60 drug-screening panel. Cancer Research 2003, 63:8634-8647.

10. Ikediobi ON, Davies H, Bignell G, Edkins S, Stevens C, O'Meara S, Santarius T, Avis T, Barthorpe S, Brackenbury L, Buck G, Butler A, Clements J, Cole J, Dicks E, Forbes S, Gray K, Halliday K, Harrison R, Hills K, Hinton J, Hunter C, Jenkinson A, Jones D, Kosmidou V, Lugg R, Menzies A, Mironenko T, Parker A, Perry J, Raine K, Richardson D, Shepherd R, Small A, Smith R, Solomon H, Stephens P, Teague J, Tofts C, Varian J, Webb T, West S, Widaa S, Yates A, Reinhold W, Weinstein JN, Stratton MR, Futreal PA, Wooster R: Mutation analysis of 24 known cancer genes in the $\mathrm{NCl}-60$ cell line set. Molecular Cancer Therapy 2006, 5(11):2606-2612.

11. Bussey KJ, Chin K, Lababidi S, Reimers M, Reinhold WC, Kuo WL, Gwadry F, Ajay, Kouros-Mehr H, Fridlyand J, Jain A, Collins C, Nishizuka S, Tonon G, Roschke A, Gehlhaus K, Kirsch I, Scudiero DA, Gray JW, Weinstein JN: Integrating data on DNA copy number with gene expression levels and drug sensitivities in the NCl-60 cell line panel. Molecular Cancer Therapy 2006, 5(4):853-867.

12. Ehrich $M$, Turner J, Gibbs $P$, Lipton L, Giovanneti $M$, Cantor C, van den Boom D: Cytosine methylation profiling of cancer cell lines. Proceedings of the National Academy of Science USA 2008, 105(12):4844-4849.

13. Blower PE, Verducci JS, Lin S, Zhou J, Chung JH, Dai Z, Liu CG, Reinhold W, Lorenzi PL, Kaldjian EP, Croce CM, Weinstein JN, Sadee W: MicroRNA expression profiles for the $\mathrm{NCl}-60$ cancer cell panel. Molecular Cancer Therapy 2007, 6(5):1483-1491.

14. Gaur A, Jewell DA, Liang Y, Ridzon D, Moore JH, Chen C, Ambros VR, Israel MA: Characterization of microRNA expression levels and their biological correlates in human cancer cell lines. Cancer Research 2007, 67(6):2456-2468

15. Ross DT, Scherf U, Eisen MB, Perou CM, Rees C, Spellman P, lyer V, Jeffrey SS, Van de Rijn M, Waltham M, Pergamenschikov A, Lee JC, Lashkari D, Shalon D, Myers TG, Weinstein JN, Botstein D, Brown PO: Systematic variation in gene expression patterns in human cancer cell lines. Nature Genetics 2000, 24:227-235.

16. Shankavaram UT, Reinhold WC, Nishizuka S, Major S, Morita D, Chary KK, Reimers MA, Scherf U, Kahn A, Dolginow D, Cossman J, Kaldjian EP, Scudiero DA, Petricoin E, Liotta L, Lee JK: Transcript and protein expression profiles of the $\mathrm{NCl}-60$ cancer cell panel: an integromic microarray study. Molecular Cancer Therapy 2007, 6(3):820-832.

17. Liu H, D'Andrade P, Fulmer-Smentek S, Lorenzi P, Kohn KW, Weinstein JN, Pommier $Y$, Reinhold WC: mRNA and microRNA expression profiles of the $\mathrm{NCl}-60$ integrated with drug activities. Molecular Cancer Therapeutics 2010, 9(5):1080-1091

18. Nishizuka S, Charboneau L, Young L, Major S, Reinhold WC, Waltham M, Kouros-Mehr H, Bussey KJ, Lee JK, Espina V, Munson PJ, Petricoin E, Liotta LA, Weinstein JN: Proteomic profiling of the $\mathrm{NCl}-60$ cancer cell lines using new high-density reverse-phase lysate microarrays. Proceedings of the National Academy of Science USA 2003, 100(24):14229-14234

19. Annereau JP, Szakacs G, Tucker CJ, Arclello A, Cardarelli C, Collins J, Grissom S, Zeeberg BR, Reinhold W, Weinstein JN, Pommier Y, Paules RS, Gottesman MM: Analysis of ATP-binding cassette transporter expression 
in drug-selected cell lines by a microarray dedicated to multidrug resistance. Molecular Pharmacology 2004, 66(6):1397-1405.

20. Leary RJ, Lin JC, Cummins J, Boca S, Wood LD, Parsons DW, Jones S, Sjoblom T, Park BH, Parsons R, Willis J, Dawson D, Willson JK, Nikolskaya T, Nikolsky Y, Kopelovich L, Papadopoulos N, Pennacchio LA, Wang TL, Markowitz SD, Parmigiani G, Kinzler KW, Vogelstein B, Velculescu VE: Integrated analysis of homozygous deletions, focal amplifications, and sequence alterations in breast and colorectal cancers. Proceedings of the National Academy of Science USA 2008, 105(42):16224-16229.

21. Subramanian A, Tamayo P, Mootha VK, Mukherjee S, Ebert BL, Gillette MA, Paulovich A, Pomeroy SL, Golub TR, Lander ES, Mesirov JP: Gene set enrichment analysis: A knowledge-based approach for interpreting genome-wide expression profiles. Proceedings of the National Academy of Science USA 2005, 102(43):15545-15550.

22. Bild AH, Yao G, Chang JT, Wang Q, Potti A, Chasse D, Joshi MB, Harpole D, Lancaster JM, Berchuck A, Olson JA Jr, Marks JR, Dressman HK, West M, Nevins JR: Oncogenic pathway signatures in human cancers as a guide to targeted therapies. Nature 2006, 439:353-357.

23. Vaske CJ, Benz SC, Sanborn JZ, Earl D, Szeto C, Zhu J, Haussler D, Stuart JM: Inference of patient-specific pathway activities from multi-dimensional cancer genomics data using PARADIGM. Bioinformatics 2010, 26(12): i237-i245.

24. Heiser LM, Wang NJ, Talcott CL, Laderoute KR, Knapp M, Guan Y, Hu Z, Ziyad S, Weber BL, Laquerre S, Jackson JR, Wooster RF, Kuo WL, Gray JW, Spellman PT: Integrated analysis of breast cancer cell lines reveals unique signaling pathways. Genome Biology 2009, 10:R31.

25. Salvesen HB, Carter SL, Mannelqvist M, Dutt A, Getz A, Stefansson IM, Raeder MB, Sos ML, Engelsen IB, Trovik J, Wik E, Greulich H, Bo TH, Jonassen I, Thomas RK, Zander T, Garraway LA, Oyan AM, Sellers WR, Kalland KH, Meyerson M, Akslen LA, Beroukhim R: Integrated genomic profiling of endometrial carcinoma associates aggressive tumors with indicators of PI3 kinase activation. Proceedings of the National Academy of Science USA 2009, 106(12):4834-4839.

26. Garraway LA, Widlund HR, Rubin MA, Getz G, Berger AJ, Ramaswamy S, Beroukhim R, Milner DA, Granter SR, Du J, Lee C, Wagner SN, Li C, Golub TR, Rimm DL, Meyerson ML, Fisher DE: Integrative genomic analyses identify MITF as a lineage survival oncogene amplified in malignant melanoma. Nature 2005, 436(7047):117-122.

27. Cifola I, Spinelli R, Beltrame L, Peano C, Fasoli E, Ferrero S, Bosari S, Signorini S, Rocco F, Perego R, Proserpio V, Raimondo F, Mocarelli P, Battaglia C: Genome-wide screening of copy number alterations and $\mathrm{LOH}$ events in renal cell carcinomas and integration with gene expression profile. Molecular Cancer 2008, 7:6.

28. Basso L, Margolin AA, Stolovitzky G, Klein U, Dalla-Favera R, Califano A: Reverse engineering of regulatory networks in human B cells. Nature Genetics 2005, 37(4):382-390.

29. Schadt EE, Lamb J, Yang X, Zhu J, Edwards S, Guhathakurta D, Sieberts SK, Monks S, Reitman M, Zhang C, Lum PY, Leonardson A, Thieringer R, Metzger JM, Yang L, Castle J, Zhu H, Kash SF, Drake TA, Sachs A, Lusis AJ: An integrative genomics approach to infer causal associations between gene expression and disease. Nature Genetics 2005, 37(7):710-717.

30. Yeang CH, Mak C, Workman C, McCuine S, Jaakkola T, Ideker T: Validation and refinement of gene-regulatory pathways on a network of physical interactions. Genome Biology 2005, 6:R62.1-R62.10.

31. Markowetz F, Bloch J, Spang R: Non-transcriptional pathway features reconstructed from secondary effects of RNA interference. Bioinformatics 2005, 21(21):4026-4032.

32. Akavia UD, Litvin O, Kim J, Sanchez-Garcia F, Kotliar D, Causton HC, Pochanard P, Mozes E, Garraway LA, Pe'er D: An integrated approach to uncover drivers of cancer. Cell 2010, 143(6):1005-1017.

33. Yeang $\mathrm{CH}$ : An integrated analysis of molecular aberrations in $\mathrm{NCl}-60$ cell lines. BMC Bioinformatics 2010, 11:495.

34. Benjamini Y, Hochberg Y: Controlling the false discovery rate: a practical and powerful approach to multiple testing. Journal of the Royal Statistical Society B 1995, 57(1):289-300.

35. Storey J, Tibshirani R: Statistical significance for genomewide studies. Proceedings of the National Academy of Science USA 2003, 100(16):9440-9445

36. Korn EL, Troendle JF, MCShane LM, Simon R: Controlling the number of false discoveries: application to high-dimensional genomic data. Journa of Statistical Planning and Inference 2004, 124:379-398.
37. Matys V, Fricke E, Geffers R, Gossling E, Haubrock M, Hehl R, Hornischer K, Karas D, Kel AE, Kel-Margoulis OV, Kloos DU, Land S, Lewicki-Potapov B, Michael H, Mnch R, Reuter I, Rotert S, Saxel H, Scheer M, Thiele S, Wingender E: TRANSFAC: transcriptional regulation, from patterns to profiles. Nucleic Acids Research 2003, 31(1):374-378.

38. Lewis BP, Burge $C B$, Bartel DP: Conserved seed pairing, often flanked by adenosines, indicates that thousands of human genes are microRNA targets. Cell 2005, 120(1):15-20.

39. Betel D, Wilson M, Gabow A, Marks D, Sander C: The microRNA.org resource: targets and expression. Nucleic Acids Research 2008, 36 D149-D153.

40. Kozomara A, Griffiths-Jones S: miRBase: integrating microRNA annotation and deep-sequencing data. Nucleic Acids Research 2011, 39:D152-D157.

41. The Gene Ontology Database:[http://www.geneontology.org/].

42. Schaefer CF, Anthony K, Krupa S, Buchoff J, Day M, Hannay T, Buetow KH: PID: the Pathway Interaction Database. Nucleic Acids Research 2009, 37 D674-679.

43. Joshi-Tope G, Gillespie M, Vastrik I, D'Eustachio P, Schmidt E, de Bono B, Jassal B, Gopinath GR, Wu GR, Matthews L, Lewis S, Birney E, Stein L: Reactome: a knowledgebase of biological pathways. Nucleic Acids Research 2005, 33:D428-432.

44. BioCarta Database:[http://www.biocarta.com/].

45. Hoek K, Schlegel NC, Eichhoff OM, Widmer DS, Praetorius C, Einarsson SO, Valgeirsdottir S, Bergsteinsdottir K, Schepsky A, Dummer R, Steingrimsson E: Novel MITF targets identified using a two-step DNA microarray strategy. Pigment Cell Melanoma Research 2008, 21:665-676.

46. Oh $I H$, Reddy EP: The myb gene family in cell growth, differentiation and apoptosis. Oncogene 1999, 18:3017-3033.

47. Lahortiga I, De Keersmaecker K, Van Vlierberghe P, Graux C, Cauwelier B, Lambert F, Mentens N, Beverloo HB, Pieters R, Speleman F, Odero MD, Bauters $M$, Froyen G, Marynen P, Vandenberghe P, Wlodarska I, Meijerink JP, Cools J: Duplication of the MYB oncogene in T cell acute lymphoblastic leukemia. Nature Genetics 2007, 39(5):593-595.

48. NCI/NIH Developmental Therapeutics Program:[http://www.dtp.nci.nih.gov].

49. Zhang SX, Garcia-Gras E, Wycuff DR, Marriot SJ, Kadeer N, Yu W, Olson EN, Garry DJ, Parmacek MS, Schwartz RJ: Identification of direct serumresponse factor gene targets during Me2SO-induced P19 cardiac cell differentiation. Journal of Biological Chemistry 2005, 280(19):19115-19126.

50. Wei CL, Wu Q, Vega VB, Chiu KP, Ng P, Zhang T, Shahab A, Yong HC, Fu Y, Weng Z, Liu J, Zhao XD, Chew JL, Lee YL, Kuznetsov VA, Sung WK, Miller LD, Lim B, Liu ET, Yu Q, Ng HH, Ruan Y: A globalMap of p53 transcription-factor binding sites in the human genome. Cell 2006, 124:207-219.

51. Vazquez A, Grochola LF, Bond EE, Levine AJ, Taubert H, Muller TH, Wurl P, Bond GL: Chemosensitivity profiles identify polymorphisms in the p53 network genes 14-3-3tau and CD44 that affect sarcoma incidence and survival. Cancer Research 2010, 70(1):172-180.

52. Mirza A, Wu Q, Wang L, McClanahan T, Bishop WR, Gheyas F, Ding W, Hutchins B, Hockenberry T, Kirschmeier P, Greene JR, Liu S: Global transcriptional program of p53 target genes during the process of apoptosis and cell cycle progression. Oncogene 2003, 22(23):3645-3654.

53. Amundson SA, Do KT, Vinikoor LC, Lee RA, Koch-Paiz CA, Ahn J, Reimers M, Chen Y, Scudiero DA, Weinstein JN, Trent JM, Bittner ML, Meltzer PS, Fornace AJ Jr: Integrating global gene expression and radiation survival parameters across the 60 cell lines of the National Cancer Institute anticancer drug screen. Cancer Research 2008, 68(2):415-424.

54. Su Al, Wiltshire T, Batalov S, Lapp H, Ching KA, Block D, Zhang J, Soden R, Hayakawa M, Kreiman G, Cooke MP, Walker JR, Hogenesch JB: A gene atlas of the mouse and human protein-encoding transcriptomes. Proceedings of National Academy of Sciences USA 2004, 101(16):6062-6067.

55. Kuhn RM, Karolchik D, Zweig AS, Wang T, Smith KE, Rosenbloom KR, Rhead B, Raney BJ, Pohl A, Pheasant M, Meyer L, Hsu F, Hinrichs AS, Harte RA, Giardine B, Fujita P, Diekhans M, Dreszer T, Clawson H, Barber GP, Haussler D, Kent WJ: The UCSC Genome Browser Database: update 2009. Nucleic Acids Research 2009, D755-D761.

doi:10.1186/1752-0509-5-186

Cite this article as: Li et al.: Deciphering causal and statistical relations of molecular aberrations and gene expressions in $\mathrm{NCl}-60$ cell lines. BMC Systems Biology 2011 5:186. 